Article

\title{
Tsallis Entropy of Fuzzy Dynamical Systems ${ }^{\dagger}$
}

\section{Dagmar Markechová}

Department of Mathematics, Faculty of Natural Sciences, Constantine the Philosopher University in Nitra, A. Hlinku 1, SK-949 01 Nitra, Slovakia; dmarkechova@ukf.sk; Tel.: +421-376-408-111; Fax: +421-376-408-020

† Dedicated to the Memory of Prof. RNDr. Beloslav Riečan, DrSc., Dr.h.c.

Received: 1 November 2018; Accepted: 14 November 2018; Published: 18 November 2018

\begin{abstract}
This article deals with the mathematical modeling of Tsallis entropy in fuzzy dynamical systems. At first, the concepts of Tsallis entropy and Tsallis conditional entropy of order $q$, where $q$ is a positive real number not equal to 1, of fuzzy partitions are introduced and their mathematical behavior is described. As an important result, we showed that the Tsallis entropy of fuzzy partitions of order $q>1$ satisfies the property of sub-additivity. This property permits the definition of the Tsallis entropy of order $q>1$ of a fuzzy dynamical system. It was shown that Tsallis entropy is an invariant under isomorphisms of fuzzy dynamical systems; thus, we acquired a tool for distinguishing some non-isomorphic fuzzy dynamical systems. Finally, we formulated a version of the Kolmogorov-Sinai theorem on generators for the case of the Tsallis entropy of a fuzzy dynamical system. The obtained results extend the results provided by Markechová and Riečan in Entropy, 2016, 18, 157, which are particularized to the case of logical entropy.
\end{abstract}

Keywords: fuzzy set; fuzzy partition; Tsallis entropy; conditional Tsallis entropy; fuzzy dynamical system; isomorphism; generators

\section{Introduction}

The concept of entropy to ergodic theory was introduced by Kolmogorov [1] and Sinai [2] in relation to the problem of isomorphisms of dynamical systems. Let $(X, S, \mu, U)$ be a dynamical system, i.e., let $(X, S, \mu)$ be a probability space and $U: X \rightarrow X$ be a measure $\mu$ preserving transformation (i.e., $G \in S$ implies $U^{-1}(G) \in S$, and $\left.\mu\left(U^{-1}(G)\right)=\mu(G)\right)$. If $\mathcal{G}=\left\{G_{1}, G_{2}, \ldots, G_{k}\right\}$ is a measurable partition of a probability space $(X, S, \mu)$ with probabilities $p_{i}=\mu\left(G_{i}\right), i=1,2, \ldots, k$, then the entropy of the partition $\mathcal{G}$ is defined as the number $H(\mathcal{G})=\sum_{i=1}^{k} F\left(p_{i}\right)$, where $F:[0,1] \rightarrow[0, \infty)$ is the Shannon entropy function defined by $F(x)=-x \cdot \log x$, if $x>0$, and $F(0)=0$ (cf. [3]). If $\mathcal{G}$ and $\mathcal{H}$ are two measurable partitions of $(X, S, \mu)$, then the family $\mathcal{G} \vee \mathcal{H}=\{G \cap H ; G \in \mathcal{G}, H \in \mathcal{H}\}$ is a measurable partition of $(X, S, \mu)$. Evidently, the family $U^{-1}(\mathcal{G})=\left\{U^{-1}(G) ; G \in \mathcal{G}\right\}$ is also a measurable partition of $(X, S, \mu)$. The Kolmogorov-Sinai entropy of a dynamical system $(X, S, \mu, U)$ is defined as the number $h(U)=\sup \{h(\mathcal{G}, U)\}$, where the supremum is taken over all finite measurable partitions $\mathcal{G}$ of probability space $(X, S, \mu)$, and $h(\mathcal{G}, U)$ is defined as $\lim _{n \rightarrow \infty} \frac{1}{n} H\left(\vee_{i=0}^{n-1} U^{-i}(\mathcal{G})\right)$. Using the proposed concept, Kolmogorov and Sinai showed the existence of non-isomorphic Bernoulli shifts.

The Kolmogorov-Sinai entropy has proved to be widely applicable. It is used to measure the complexity of the motion in a dynamical system; Russian mathematician Pesin (cf. [4]) demonstrated that when the Kolmogorov-Sinai entropy is greater than zero, the dynamical system will display chaos. Successful applications of Kolmogorov-Sinai entropy of a dynamical system stimulated the study of alternative entropy measures of dynamical systems. We note that in Reference [5], the notion of logical entropy $h_{L}(U)$ of a dynamical system $(X, S, \mu, U)$ was proposed. It has turned out that if the Shannon 
entropy function $F$ is replaced by the function $L:[0,1] \rightarrow[0, \infty)$ defined, for every $x \in[0,1]$, by the following equation:

$$
L(x)=x-x^{2},
$$

the results analogous to the case of Kolmogorov-Sinai entropy theory are obtained. The logical entropy $h_{L}(U)$ is invariant under isomorphisms of dynamical systems; therefore, it can be used as an alternative tool for distinguishing some non-isomorphic dynamical systems. We note that some other recently published results concerning the logical entropy can be found, for example, in References [6-17].

In Reference [9], the logical entropy of fuzzy dynamical systems was studied. We remind the reader that fuzzy set theory was introduced by Zadeh in Reference [18] as an extension of the classical Cantor set theory. While in the classical set theory, the membership of elements in a set is assessed in binary terms according to a bivalent condition-an element either belongs or does not belong to the set, fuzzy set theory allows the assessment of the membership of elements in a set. This is described by a membership function which assigns to every element a degree of membership ranging in the real unit interval $[0,1]$. Fuzzy sets are a generalization of classical sets, because the characteristic functions of classical sets are special cases of the membership functions of fuzzy sets. Fuzzy set theory can be used in a wide range of domains in which information is incomplete or imprecise. Since the seminal publication [18], fuzzy set theory has advanced in various mathematical disciplines and it has also found many significant practical applications-for example, in control theory, data analysis, artificial intelligence, and computational intelligence. Of course, many publications (see e.g., [19-33]) are devoted to the study of entropy in the fuzzy case. In our work [9], we introduced the concept of logical entropy of fuzzy dynamical systems. Instead of measurable partitions, we considered so-called fuzzy partitions that can be used for modeling experiments with vague, incomplete information. The aim of this paper is to generalize the results regarding the logical entropy in the fuzzy case given in Reference [9] to the case of Tsallis entropy.

Tsallis entropy, as a generalization of standard Shannon-type entropy, was introduced by Constantino Tsallis in Reference [34]. Since then, the concept has been extensively studied. Tsallis entropy in its form is identical with Havrda-Charvát alpha-entropy, introduced in Reference [35] in the framework of information theory. If $P=\left\{p_{1}, p_{2}, \ldots, p_{n}\right\}$ is a probability distribution, then its Tsallis entropy of order $q$, where $q \in(0,1) \cup(1, \infty)$, is defined by the following equation:

$$
T_{q}(P)=\frac{1}{q-1}\left(1-\sum_{i=1}^{n} p_{i}{ }^{q}\right) .
$$

Tsallis entropy plays a significant role in the non-extensive statistical mechanics of complex systems [36]. The number $q$ is the so-called entropic index; it characterizes the degree of non-extensivity of the system. Applications of Tsallis entropy have been found for a wide range of phenomena in diverse disciplines such as chemistry, physics, geophysics, biology, economics, medicine, etc. (see, e.g., [37-49]). Tsallis entropy is also applicable to large domains in communication systems (cf. [50]); its image processing applications through information theory can be found, for example, in Reference [51]. For a full and regularly updated bibliography, see Reference [52].

Let us define, for any real number $q \neq 1$, the function $L_{q}:[0,1] \rightarrow[0, \infty)$ by equation:

$$
L_{q}(x)=\frac{1}{q-1}\left(x-x^{q}\right),
$$

for every $x \in[0,1]$. Obviously, for $q \in(0,1) \cup(1, \infty)$, we can write:

$$
T_{q}(P)=\sum_{i=1}^{n} L_{q}\left(p_{i}\right) .
$$


If we put $q=2$ into Equation (4), then we obtain:

$$
T_{2}(P)=\sum_{i=1}^{n} L_{2}\left(p_{i}\right)=\sum_{i=1}^{n}\left(p_{i}-p_{i}{ }^{2}\right)=1-\sum_{i=1}^{n} p_{i}{ }^{2},
$$

which is the logical entropy of the probability distribution $P=\left\{p_{1}, p_{2}, \ldots, p_{n}\right\}$ studied in Reference [6]. The logical entropy of partitions in a product MV-algebra (cf. [53-55]) was defined and studied in Reference [10]. Another recently published paper [56] was devoted to the mathematical modeling of Tsallis entropy in product MV-algebra dynamical systems. It should be noted that the full tribe of fuzzy sets represents a special case of product MV-algebras; therefore, the results provided in References $[10,56]$ can be immediately applied to this important case of fuzzy sets. It is known that there are many possibilities for defining operations over fuzzy sets; for an overview, see Reference [57]. While in the case of the full tribe of fuzzy sets, Łukasiewicz connectives were applied, the access in this paper is based on standard Zadeh connectives [18].

The presentation in this paper is structured as follows. Section 2 provides definitions, notations, and some known facts used in the article. In Section 3, the concept of the Tsallis entropy of order $q \in$ $(0,1) \cup(1, \infty)$ of a fuzzy partition and its conditional version are introduced and studied. It is shown that the proposed definitions of Tsallis entropies generalize the logical entropy of fuzzy partitions studied in Reference [9]; it is enough to put $q=2$. In addition, they are consistent, in the case of the limit of $q \rightarrow 1$, with the Shannon-type entropy of fuzzy partitions proposed in Reference [29] (see also [30,31]). Section 4 deals with the mathematical modeling of the Tsallis entropy of fuzzy dynamical systems. Using the proposed notion of Tsallis entropy of a fuzzy partition, we define the Tsallis entropy of order $q>1$ of a fuzzy dynamical system. It turns out that the Tsallis entropy is an invariant under isomorphisms of fuzzy dynamical systems, and can thus be used as a tool for distinguishing non-isomorphic fuzzy dynamical systems. Finally, we formulate a version of the Kolmogorov-Sinai theorem on generators for the case of the Tsallis entropy of a fuzzy dynamical system. The last section provides brief closing remarks.

\section{Basic Definitions, Notations, and Facts}

Let us start with recalling the basic terms and some of the known results that are used in the article.

It is known that the classical Cantor set $A$ in the universe $X$ can be represented by the characteristic function $\varphi_{A}$ mapping $X$ into the set $\{0,1\}$, namely, for $x \in X, \varphi_{A}(x)=0$, if $x \notin A$, and $\varphi_{A}(x)=1$, if $x \in A$. A fuzzy subset of $X$ is defined by a membership function mapping $X$ into the unit interval $[0,1]$ : by a fuzzy subset of $X$, we understand a map $a: X \rightarrow[0,1]$ (where the considered fuzzy set is identified with its membership function). The value $a(x)$ is considered as a degree of membership of the element $x \in X$ to the fuzzy set $a$. If $a(x)=0$, then $x$ does not belong to $a$, if $a(x)=1$, then $x$ belongs to $a$, and if $a(x) \in(0,1)$, then $x$ possibly belongs to $a$, but this is not certain. For the last case, the nearer to 1 the value $a(x)$ is, the higher the possibility that $x$ belongs to $a$. Let $X$ be a non-empty set. By the symbols $\cup_{n=1}^{\infty} a_{n}$ and $\cap_{n=1}^{\infty} a_{n}$, we denote the fuzzy union and the fuzzy intersection of a sequence $\left\{a_{n}\right\}_{n=1}^{\infty}$ of fuzzy subsets of $X$, respectively, in the sense of Zadeh [18]; i.e., $\cup_{n=1}^{\infty} a_{n}=\sup _{n} a_{n}$, and $\cap_{n=1}^{\infty} a_{n}=\inf _{n} a_{n}$. The symbol $a^{\perp}$ denotes the complement of fuzzy subset $a$ of $X$, i.e., $a^{\perp}=1_{X}-a$. Here, $1_{X}$ indicates the constant function with the value 1 . Analogously, the symbols $(1 / 2)_{X}$ and $0_{X}$ will indicate the constant functions with the value $1 / 2$, and 0 , respectively. Additionally, the relation $\leq$ will indicate the usual order relation of fuzzy subsets of $X$, i.e., $a \leq b$ if and only if $a(x) \leq b(x)$, for every $x \in X$. The complementation $\perp: a \rightarrow a^{\perp}$ satisfies, for every fuzzy subset $a, b$ of $X$, the conditions: $\left(a^{\perp}\right)^{\perp}=a$, and $a \leq b$ implies $b^{\perp} \leq a^{\perp}$. Further, for any sequence $\left\{a_{n}\right\}_{n=1}^{\infty}$ of fuzzy subsets of $X$, the de Morgan laws hold: $\left(\cup_{n=1}^{\infty} a_{n}\right)^{\perp}=\cap_{n=1}^{\infty} a_{n}^{\perp}$, and $\left(\cap_{n=1}^{\infty} a_{n}\right)^{\perp}=\cup_{n=1}^{\infty} a_{n}^{\perp}$.

A fuzzy measurable space [58] is a couple $(X, M)$, where $X$ is a non-empty set, and $M$ is a fuzzy $\sigma$-algebra of fuzzy subsets of $X$, i.e., $M \subset[0,1]^{X}$ containing $1_{X}$, excluding $(1 / 2)_{X}$, closed under the operation $\perp$ (i.e., if $a \in M$, then $a^{\perp} \in M$ ) and countable supremums (i.e., satisfying the implication 
if $a_{n} \in M, n=1,2, \ldots$, then $\left.\cup_{n=1}^{\infty} a_{n} \in M\right)$. It can be verified that the concept of a fuzzy measurable space generalizes the notion of a measurable space $(X, S)$ from the classical measure theory; it is enough to put $M=\left\{\varphi_{A} ; A \in S\right\}$, where $\varphi_{A}$ is the characteristic function of the set $A \in S$. Using this procedure, the classical model can be embedded into the fuzzy case. Fuzzy sets $a, b \in M$ such that $a \cap b=0_{X}$ are considered to be separated, while fuzzy sets $a, b \in M$ such that $a \leq b^{\perp}$ are considered to be W-separated [59]. A fuzzy set $a \in M$ such that $a \geq a^{\perp}$ is called a W-universum; a fuzzy set $a \in M$ such that $a \leq a^{\perp}$ is called a W-empty fuzzy set. It can be proved that a fuzzy set $a \in M$ is a $\mathrm{W}$-universum if and only if there exists a fuzzy set $b \in M$ such that $a=b \cup b^{\perp}$. The following definition was introduced in Reference [60].

Definition 1 [60]. Let $(X, M)$ be a fuzzy measurable space. A map $s: M \rightarrow[0,1]$ is called a fuzzy P-measure, if the following two conditions are satisfied: (i) $s\left(a \cup a^{\perp}\right)=1$, for every $a \in M$; (ii) if $\left\{a_{n}\right\}_{n=1}^{\infty}$ is a sequence of pairwise $W$-separated fuzzy subsets from $M$, then $s\left(\cup_{n=1}^{\infty} a_{n}\right)=\sum_{n=1}^{\infty} s\left(a_{n}\right)$. The triplet $(X, M, s)$ is said to be a fuzzy probability space.

A fuzzy subset belonging to the fuzzy $\sigma$-algebra $M$ is regarded as a fuzzy event; W-separated fuzzy events are regarded as mutually exclusive events. A $\mathrm{W}$-universum is considered as a certain event, while a W-empty set as an impossible event. The fuzzy P-measure $s: M \rightarrow[0,1]$ has properties analogous to properties of a classical probability measure (for the proof, see [60]). We present some of them below.

(P1) $s\left(a^{\perp}\right)=1-s(a)$, for every $a \in M$.

(P2) $s$ is non-decreasing, i.e., if $a, b \in M$ with $a \leq b$, then $s(a) \leq s(b)$.

(P3) $s(a \cup b)+s(a \cap b)=s(a)+s(b)$, for every $a, b \in M$.

(P4) Let $b \in M$. Then $s(a \cap b)=s(a)$ for all $a \in M$ if and only if $s(b)=1$.

(P5) If $a, b \in M$ such that $a \leq b^{\perp}$, then $s(a \cap b)=0$.

Definition 2. Let $(X, M, s)$ be a fuzzy probability space. If $a, b \in M$, then we define:

$$
s(a / b)= \begin{cases}\frac{s(a \cap b)}{s(b)}, & \text { if } f_{s}(b)>0 \\ 0, & \text { if } f_{s}(b)=0 .\end{cases}
$$

Let $s: M \rightarrow[0,1]$ be a fuzzy P-measure, and let $b \in M$ such that $s(b)>0$. Then the map $s(\cdot / b): M \rightarrow[0,1]$ defined by Equation (5) is a fuzzy P-measure. It plays the role of a conditional probability measure on the family $M$ of fuzzy events. The following definition of a fuzzy partition was introduced in Reference [61].

Definition 3 [61]. A fuzzy partition of a fuzzy probability space $(X, M, s)$ is a family $\alpha=\left\{a_{1}, a_{2}, \ldots, a_{n}\right\}$ of pairwise $W$-separated fuzzy sets from $M$ with the property $s\left(\cup_{i=1}^{n} a_{i}\right)=1$.

In the class of all fuzzy partitions of fuzzy probability space $(X, M, s)$, we define the refinement partial order as follows. If $\alpha=\left\{a_{1}, a_{2}, \ldots, a_{k}\right\}$ and $\beta=\left\{b_{1}, b_{2}, \ldots, b_{l}\right\}$ are two fuzzy partitions of $(X, M, s)$, then we say that $\beta$ is a refinement of $\alpha$ (and write $\alpha \prec \beta$ ), if there exists a partition $\left\{I_{1}, I_{2}, \ldots, I_{k}\right\}$ of the set $\{1,2, \ldots, l\}$ such that $a_{i}=\cup_{j \in I_{i}} b_{j}$, for $i=1,2, \ldots, k$. Further, we set $\alpha \vee \beta=\left\{a_{i} \cap b_{j} ; i=1,2, \ldots, k, j=1,2, \ldots, l\right\}$. One can easily to verify that the family $\alpha \vee \beta$ is a family of pairwise $\mathrm{W}$-separated fuzzy sets from $M$; moreover, by the property (P4), we obtain $s\left(\cup_{i=1}^{k} \cup_{j=1}^{l}\left(a_{i} \cap b_{j}\right)\right)=s\left(\left(\cup_{i=1}^{k} a_{i}\right) \cap\left(\cup_{j=1}^{l} b_{j}\right)\right)=s\left(\cup_{i=1}^{k} a_{i}\right)=1$. This means that $\alpha \vee \beta$ is a fuzzy partition of $(X, M, s)$; it represents a combined experiment consisting of a realization of the experiments $\alpha$ and $\beta$. If $\alpha_{1}, \alpha_{2}, \ldots, \alpha_{n}$ are fuzzy partitions of $(X, M, s)$, then we put $\vee_{i=1}^{n} \alpha_{i}=\alpha_{1} \vee \alpha_{2} \vee \ldots \vee \alpha_{n}$. 
Example 1. Let $(X, S, \mu)$ be a classical probability space. If we set $M=\left\{\varphi_{A} ; A \in S\right\}$, where $\varphi_{A}$ is the characteristic function of the set $A \in S$, and define the map $s: M \rightarrow[0,1]$ by the formula $s\left(\varphi_{A}\right)=\mu(A)$, for every $\varphi_{A} \in M$, then it is easy to verify that the system $(X, M, s)$ is a fuzzy probability space. A measurable partition $G=\left\{G_{1}, G_{2}, \ldots, G_{k}\right\}$ of probability space $(X, S, \mu)$ can be viewed as a fuzzy partition if we consider $\varphi_{G_{i}}$ instead of $G_{i}$.

Definition 4. Two fuzzy partitions $\alpha=\left\{a_{1}, a_{2}, \ldots, a_{k}\right\}$ and $\beta=\left\{b_{1}, b_{2}, \ldots, b_{l}\right\}$ of a fuzzy probability space $(X, M, s)$ are said to be statistically independent if $s\left(a_{i} \cap b_{j}\right)=s\left(a_{i}\right) \cdot s\left(b_{j}\right)$, for $i=1,2, \ldots, k, j=1,2, \ldots, l$.

The following definition of logical entropy and conditional logical entropy of fuzzy partitions was introduced in Reference [9].

Definition 5 ([9]). Let $\alpha=\left\{a_{1}, a_{2}, \ldots, a_{k}\right\}, \beta=\left\{b_{1}, b_{2}, \ldots, b_{l}\right\}$ be two fuzzy partitions of a fuzzy probability space $(X, M, s)$. We define the logical entropy of $\alpha$ by:

$$
H_{L}(\alpha)=\sum_{i=1}^{k} s\left(a_{i}\right)\left(1-s\left(a_{i}\right)\right)
$$

The conditional logical entropy of $\alpha$ given $\beta$ is defined by the following equation:

$$
H_{L}(\alpha / \beta)=\sum_{i=1}^{k} \sum_{j=1}^{l} s\left(a_{i} \cap b_{j}\right)\left(s\left(b_{j}\right)-s\left(a_{i} \cap b_{j}\right)\right) .
$$

Remark 1. It is evident that we can write Equation (6) in the form $H_{L}(\alpha)=\sum_{i=1}^{k} L\left(s\left(a_{i}\right)\right)$, where $L:[0,1] \rightarrow[0, \infty)$ is the logical entropy function defined by Equation (1). Equation (7) can be expressed in the following form:

$$
H_{L}(\alpha / \beta)=\sum_{j=1}^{l}\left(s\left(b_{j}\right)\right)^{2}-\sum_{i=1}^{k} \sum_{j=1}^{l}\left(s\left(a_{i} \cap b_{j}\right)\right)^{2}
$$

In Reference [9], we proved the basic properties of the suggested entropy measures. Specifically, the logical entropy of fuzzy partitions was shown to have the property of sub-additivity (i.e., $H_{L}(\alpha \vee$ $\beta) \leq H_{L}(\alpha)+H_{L}(\beta)$, for arbitrary fuzzy partitions $\alpha, \beta$ of $\left.(X, M, s)\right)$, and not to have the property of additivity. It satisfies the following weaker property: if fuzzy partitions $\alpha, \beta$ of $(X, M, s)$ are statistically independent, then $1-H_{L}(\alpha \vee \beta)=\left(1-H_{L}(\alpha)\right) \cdot\left(1-H_{L}(\beta)\right)$. The definition of the Shannon-type entropy of fuzzy partitions was proposed in Reference [29], and is given as follows.

Definition 6 ([29]). Let $\alpha=\left\{a_{1}, a_{2}, \ldots, a_{k}\right\}, \beta=\left\{b_{1}, b_{2}, \ldots, b_{l}\right\}$ be two fuzzy partitions of a fuzzy probability space $(X, M, s)$. We define the entropy of $\alpha$ by:

$$
H_{s}(\alpha)=-\sum_{i=1}^{k} s\left(a_{i}\right) \cdot \log s\left(a_{i}\right)
$$

The conditional entropy of $\alpha$ given $b_{j} \in \beta$ is defined by the following equation:

$$
H_{s}\left(\alpha / b_{j}\right)=-\sum_{i=1}^{k} s\left(a_{i} / b_{j}\right) \cdot \log s\left(a_{i} / b_{j}\right)
$$

The conditional entropy of $\alpha$ given $\beta$ is defined by the following equation:

$$
H_{s}(\alpha / \beta)=\sum_{j=1}^{l} s\left(b_{j}\right) H_{s}\left(\alpha / b_{j}\right)=-\sum_{i=1}^{k} \sum_{j=1}^{l} s\left(a_{i} \cap b_{j}\right) \cdot \log \frac{s\left(a_{i} \cap b_{j}\right)}{s\left(b_{j}\right)} \text {. }
$$

In Definition 6, it is assumed that $0 \cdot \log \frac{0}{x}=0$, if $x \geq 0$. The base of the logarithm can be any positive real number; depending on the selected base $b$ of the logarithm, information is measured in bits 
$(b=2)$, nats $(b=e)$, or dits $(b=10)$. In Reference [29], it was shown that the proposed entropy of fuzzy partitions has properties analogous to the properties of Shannon's entropy of classical measurable partitions. Specifically, for any fuzzy partitions $\alpha, \beta$ of a fuzzy probability space $(X, M, s)$, it holds $H_{s}(\alpha \vee \beta) \leq H_{s}(\alpha)+H_{s}(\beta)$ with equality if and only if the fuzzy partitions $\alpha, \beta$ are statistically independent. This means that the Shannon-type entropy of fuzzy partitions has the sub-additivity property as well as the additivity property.

In the succeeding sections, we will use the following known Jensen inequality: for a real concave function $\varphi$, real numbers $x_{1}, x_{2}, \ldots, x_{n}$ in its domain and non-negative real numbers $\lambda_{1}, \lambda_{2}, \ldots, \lambda_{n}$ satisfying the condition $\sum_{k=1}^{n} \lambda_{k}=1$, it holds that:

$$
\varphi\left(\sum_{k=1}^{n} \lambda_{k} x_{k}\right) \geq \sum_{k=1}^{n} \lambda_{k} \varphi\left(x_{k}\right)
$$

and the inequality is reversed if $\varphi$ is a real convex function. The equality in Equation (10) holds if and only if $x_{1}=x_{2}=\ldots=x_{n}$ or $\varphi$ is a linear function.

\section{The Tsallis Entropy of Fuzzy Partitions}

In this section, we define and study the Tsallis entropy of fuzzy partitions and its conditional version. Here, we assume that $(X, M, s)$ is a fuzzy probability space.

Definition 7. Let $\alpha=\left\{a_{1}, a_{2}, \ldots, a_{n}\right\}$ be a fuzzy partition of $(X, M, s)$. Then its Tsallis entropy of order $q$, where $q \in(0,1) \cup(1, \infty)$, with respect to $s$ is defined by the following equation:

$$
T_{q}^{s}(\alpha)=\frac{1}{q-1}\left(1-\sum_{k=1}^{n} s\left(a_{k}\right)^{q}\right)
$$

Remark 2. For simplicity, we write $s\left(a_{k}\right)^{q}$ instead of $\left(s\left(a_{k}\right)\right)^{q}$.

Definition 8. Let $\alpha=\left\{a_{1}, a_{2}, \ldots, a_{n}\right\}$ and $\beta=\left\{b_{1}, b_{2}, \ldots, b_{m}\right\}$ be two fuzzy partitions of $(X, M, s)$. We define the conditional Tsallis entropy of order $q$, where $q \in(0,1) \cup(1, \infty)$, of $\alpha$ given $\beta$ as the number:

$$
T_{q}^{s}(\alpha / \beta)=\frac{1}{q-1}\left(\sum_{j=1}^{m} s\left(b_{j}\right)^{q}-\sum_{i=1}^{n} \sum_{j=1}^{m} s\left(a_{i} \cap b_{j}\right)^{q}\right) .
$$

Example 2. Let us consider the fuzzy partition $\varepsilon=\{b\}$, where $b \in M$ is a W-universum. It represents a fuzzy experiment resulting in a certain event. Evidently, $T_{q}^{s}(\varepsilon)=L_{q}(s(b))=L_{q}(1)=0$, i.e., the fuzzy experiment, the outcome of which is a certain event, has zero Tsallis entropy. Furthermore, for every fuzzy partition $\alpha=\left\{a_{1}, a_{2}, \ldots, a_{n}\right\}$ of $(X, M, s)$, due to property (P4), we obtain:

$$
T_{q}^{s}(\alpha / \varepsilon)=\frac{1}{q-1}\left(s(b)^{q}-\sum_{k=1}^{n} s\left(a_{k} \cap b\right)^{q}\right)=\frac{1}{q-1}\left(1-\sum_{k=1}^{n} s\left(a_{k}\right)^{q}\right)=T_{q}^{s}(\alpha) .
$$

Let $L_{q}:[0,1] \rightarrow[0, \infty)$ be the function defined by Equation (3). Then we can write Equation (11) in the following equivalent form:

$$
T_{q}^{s}(\alpha)=\sum_{k=1}^{n} L_{q}\left(s\left(a_{k}\right)\right) .
$$

It can be verified that the function $L_{q}$ is, for every $q \in(0,1) \cup(1, \infty)$, concave and non-negative. The non-negativity of the function $L_{q}$ (for the proof, see [56]) implies that the Tsallis entropy is always non-negative. Obviously, by inserting $q=2$ into Equation (11), we obtain the logical entropy $H_{L}(\alpha)$. 
If we insert $q=2$ into Equation (12), we obtain the conditional logical entropy $H_{L}(\alpha / \beta)$. In addition, as a limiting case for $q \rightarrow 1$, we obtain the Shannon entropy of fuzzy partitions expressed in nats, as shown by the following theorems. In the proofs, we will need the following propositions.

Proposition 1. Let $\alpha=\left\{a_{1}, a_{2}, \ldots, a_{n}\right\}$ be a fuzzy partition of a fuzzy probability space $(X, M, s)$. Then

(i) $\sum_{k=1}^{n} s\left(a_{k} \cap b\right)=s(b)$, for every $b \in M$;

(ii) $\sum_{k=1}^{n} s\left(a_{k} / b\right)=1$, for every $b \in M$ such that $s(b)>0$.

Proof. The claim (i) is obtained using the condition (P4). If $b \in M$ such that $s(b)>0$, then using the part (i), we obtain:

$$
\sum_{k=1}^{n} s\left(a_{k} / b\right)=\frac{1}{s(b)} \sum_{k=1}^{n} s\left(a_{k} \cap b\right)=\frac{s(b)}{s(b)}=1
$$

Proposition 2. Let $\alpha, \beta, \gamma, \delta$ be fuzzy partitions of $(X, M, s)$. Then:

(i) $\quad \alpha \prec \beta$ implies $\alpha \vee \gamma \prec \beta \vee \gamma$;

(ii) $\quad \alpha \prec \beta$ and $\gamma \prec \delta$ implies $\alpha \vee \gamma \prec \beta \vee \delta$.

Proof. Let $\alpha=\left\{a_{1}, a_{2}, \ldots, a_{k}\right\}, \beta=\left\{b_{1}, b_{2}, \ldots, b_{l}\right\}, \gamma=\left\{c_{1}, c_{2}, \ldots, c_{m}\right\}, \delta=\left\{d_{1}, d_{2}, \ldots, d_{n}\right\}$.

(i) Let us suppose that $\alpha \prec \beta$. Then there exists a partition $\left\{I_{1}, I_{2}, \ldots, I_{k}\right\}$ of the set $\{1,2, \ldots, l\}$ such that $a_{i}=\cup_{j \in I_{i}} b_{j}$, for $i=1,2, \ldots, k$. Set $I_{i, r}=\left\{(j, r) ; j \in I_{i}\right\}$, for $i=1,2, \ldots, k, r=1,2, \ldots, m$. We then obtain:

$$
a_{i} \cap c_{r}=\left(\cup_{j \in I_{i}} b_{j}\right) \cap c_{r}=\cup_{j \in I_{i}}\left(b_{j} \cap c_{r}\right)=\cup_{(j, t) \in I_{i, r}}\left(b_{j} \cap c_{t}\right),
$$

for $i=1,2, \ldots, k, r=1,2, \ldots, m$, which means that $\alpha \vee \gamma \prec \beta \vee \gamma$.

(ii) Let us suppose that $\alpha \prec \beta$ and $\gamma \prec \delta$. Then there exists a partition $\left\{I_{1}, I_{2}, \ldots, I_{k}\right\}$ of the set $\{1,2, \ldots, l\}$ such that $a_{i}=\cup_{j \in I_{i}} b_{j}$, for $i=1,2, \ldots, k$, and there exists a partition $\left\{J_{1}, J_{2}, \ldots, J_{m}\right\}$ of the set $\{1,2, \ldots, n\}$ such that $c_{r}=\cup_{t \in J_{r}} d_{t}$, for $r=1,2, \ldots, m$. Set $K_{i, r}=\left\{(j, t) ; j \in I_{i}, t \in J_{r}\right\}$, for $i=1,2, \ldots, k, r=1,2, \ldots, m$. We thus obtain:

$$
a_{i} \cap c_{r}=\left(\cup_{j \in I_{i}} b_{j}\right) \cap\left(\cup_{t \in J_{r}} d_{t}\right)=\cup_{j \in I_{i}} \cup_{t \in J_{r}}\left(b_{j} \cap d_{t}\right)=\cup_{(j, t) \in K_{i, r}}\left(b_{j} \cap d_{t}\right),
$$

for $i=1,2, \ldots, k, r=1,2, \ldots, m$, which means that $\alpha \vee \gamma \prec \beta \vee \delta$.

Theorem 1. Let $\alpha=\left\{a_{1}, a_{2}, \ldots, a_{n}\right\}$ and $\beta=\left\{b_{1}, b_{2}, \ldots, b_{m}\right\}$ be two fuzzy partitions of $(X, M, s)$. Then:

$$
\lim _{q \rightarrow 1} T_{q}^{s}(\alpha / \beta)=-\sum_{i=1}^{n} \sum_{j=1}^{m} s\left(a_{i} \cap b_{j}\right) \cdot \ln \frac{s\left(a_{i} \cap b_{j}\right)}{s\left(b_{j}\right)} .
$$

Proof. Let us define on $(0, \infty)$ the functions $f$ and $g$ by $f(q)=\sum_{j=1}^{m} s\left(b_{j}\right)^{q}-\sum_{i=1}^{n} \sum_{j=1}^{m} s\left(a_{i} \cap b_{j}\right)^{q}$, and $g(q)=q-1$. Then for any $q \in(0,1) \cup(1, \infty)$, we have $T_{q}^{s}(\alpha / \beta)=\frac{f(q)}{g(q)}$. The functions $f$ and $g$ are differentiable and, using Proposition 1, we obtain:

$$
\lim _{q \rightarrow 1} f(q)=\sum_{j=1}^{m} s\left(b_{j}\right)-\sum_{i=1}^{n} \sum_{j=1}^{m} s\left(a_{i} \cap b_{j}\right)=1-\sum_{i=1}^{n} s\left(a_{i}\right)=1-1=0 .
$$


In addition, it is evident that $\lim _{q \rightarrow 1} g(q)=0$. Therefore, we can use L'Hôpital's rule, according to which $\lim _{q \rightarrow 1} T_{q}^{s}(\alpha / \beta)=\lim _{q \rightarrow 1} \frac{f^{\prime}(q)}{g^{\prime}(q)}$, under the assumption that the right-hand side exists. To find the derivative of function $f$, we use the identity $b^{q}=e^{q \ln b}$. We thus obtain:

$$
\frac{\mathrm{d}}{\mathrm{d} q} f(q)=\sum_{j=1}^{m} \frac{\mathrm{d}}{\mathrm{d} q}\left(s\left(b_{j}\right)^{q}\right)-\sum_{i=1}^{n} \sum_{j=1}^{m} \frac{\mathrm{d}}{\mathrm{d} q}\left(s\left(a_{i} \cap b_{j}\right)^{q}\right)=\sum_{j=1}^{m} s\left(b_{j}\right)^{q} \ln s\left(b_{j}\right)-\sum_{i=1}^{n} \sum_{j=1}^{m} s\left(a_{i} \cap b_{j}\right)^{q} \cdot \ln s\left(a_{i} \cap b_{j}\right) .
$$

Since $\frac{\mathrm{d}}{\mathrm{d} q} g(q)=1$, using Proposition 1, we obtain:

$$
\begin{gathered}
\lim _{q \rightarrow 1} T_{q}^{s}(\alpha / \beta)=\lim _{q \rightarrow 1} f^{\prime}(q)=\sum_{j=1}^{m} s\left(b_{j}\right) \ln s\left(b_{j}\right)-\sum_{i=1}^{n} \sum_{j=1}^{m} s\left(a_{i} \cap b_{j}\right) \cdot \ln s\left(a_{i} \cap b_{j}\right) \\
=\sum_{j=1}^{m} \sum_{i=1}^{n} s\left(a_{i} \cap b_{j}\right) \cdot \ln s\left(b_{j}\right)-\sum_{i=1}^{n} \sum_{j=1}^{m} s\left(a_{i} \cap b_{j}\right) \cdot \ln s\left(a_{i} \cap b_{j}\right)=-\sum_{i=1}^{n} \sum_{j=1}^{m} s\left(a_{i} \cap b_{j}\right) \cdot \ln \frac{s\left(a_{i} \cap b_{j}\right)}{s\left(b_{j}\right)} .
\end{gathered}
$$

Theorem 2. Let $\alpha=\left\{a_{1}, a_{2}, \ldots, a_{n}\right\}$ be any fuzzy partition of $(X, M, s)$. Then:

$$
\lim _{q \rightarrow 1} T_{q}^{s}(\alpha)=-\sum_{k=1}^{n} s\left(a_{k}\right) \cdot \ln s\left(a_{k}\right)
$$

Proof. The claim immediately follows from Theorem 1; it is enough to set $\beta=\left\{1_{X}\right\}$.

In the following, we derive basic properties of the suggested entropy measures of fuzzy partitions. First, we show that the function $T_{q}^{s}(\alpha)$ monotonically decreases with respect to $q \in(0,1) \cup(1, \infty)$.

Theorem 3. Let $\alpha$ be a given fuzzy partition of $(X, M, s)$ and $q_{1}, q_{2}$ be positive real numbers such that $q_{1} \neq 1, q_{2} \neq 1$. Then $q_{1} \geq q_{2}$ implies $T_{q_{1}}^{s}(\alpha) \leq T_{q_{2}}^{s}(\alpha)$.

Proof. We have to prove that $\frac{\mathrm{d}}{\mathrm{d} q} T_{q}^{s}(\alpha) \leq 0$. Suppose that $\alpha=\left\{a_{1}, a_{2}, \ldots, a_{n}\right\}$. We thus obtain:

$$
\begin{gathered}
\frac{\mathrm{d}}{\mathrm{d} q} T_{q}^{s}(\alpha)=-\frac{1}{(q-1)^{2}}\left(1-\sum_{k=1}^{n} s\left(a_{k}\right)^{q}\right)-\frac{1}{q-1} \sum_{k=1}^{n} s\left(a_{k}\right)^{q} \ln s\left(a_{k}\right) \\
=\frac{1}{(1-q)^{2}}\left(-1+\sum_{k=1}^{n} s\left(a_{k}\right)^{q}+(1-q) \sum_{k=1}^{n} s\left(a_{k}\right)^{q} \ln s\left(a_{k}\right)\right)=\frac{1}{(1-q)^{2}} \sum_{k \in K} s\left(a_{k}\right)^{q} h_{q}\left(s\left(a_{k}\right)\right),
\end{gathered}
$$

where $K=\left\{k ; s\left(a_{k}\right)>0\right\}$, and the function $h_{q}$ is defined by $h_{q}(x)=1-x^{1-q}+(1-q) \ln x$, for any $x \in(0,1]$. We prove that $h_{q}(x) \leq 0$, for every $0<x \leq 1$. We find $\frac{\mathrm{d}}{\mathrm{d} x} h_{q}(x)=\frac{(1-q)\left(1-x^{1-q}\right)}{x}$. Since $\frac{\mathrm{d}}{\mathrm{d} x} h_{q}(x)=\frac{(1-q)\left(1-x^{1-q}\right)}{x} \geq 0$, for $0<x \leq 1$, it follows that $h_{q}(x) \leq h_{q}(1)=0$, for every $x \in(0,1]$. Therefore, $\frac{\mathrm{d}}{\mathrm{d} q} T_{q}^{S}(\alpha) \leq 0$.

Remark 3. As obvious consequences of Theorems 2 and 3, we have the following relations between Tsallis entropy $T_{q}^{s}(\alpha)$ and Shannon's entropy $H_{s}(\alpha): T_{q}^{s}(\alpha) \geq H_{s}(\alpha)$, for $q \in(0,1)$, and $T_{q}^{s}(\alpha) \leq H_{s}(\alpha)$, for $q \in(1, \infty)$. Of course, in the previous inequalities, the entropy $H_{s}(\alpha)$ is expressed in nats.

Example 3. Let $X=[0,1]$, and let $a:[0,1] \rightarrow[0,1]$ be a fuzzy subset of $X$ defined by $a(x)=x$, for every $x \in[0,1]$. Then the couple $(X, M)$, where $M=\left\{a, a^{\perp}, a \cup a^{\perp}, a \cap a^{\perp}, 1_{X}, 0_{X}\right\}$, is a fuzzy measurable space. We define the fuzzy P-measure $s: M \rightarrow[0,1]$ by the equalities $s(a)=\frac{1}{3}, s\left(a^{\perp}\right)=\frac{2}{3}, s\left(1_{X}\right)=s\left(a \cup a^{\perp}\right)=1$, $s\left(0_{X}\right)=s\left(a \cap a^{\perp}\right)=0$. The families $\alpha=\left\{a, a^{\perp}\right\}, \beta=\left\{a \cup a^{\perp}\right\}, \gamma=\left\{1_{X}\right\}$ are fuzzy partitions of the fuzzy probability space $(X, M, s)$. Evidently, $T_{q}^{s}(\beta)=T_{q}^{s}(\gamma)=0$. Let us calculate entropies of the fuzzy 
partition $\alpha$. Elementary calculations show that it has a Shannon entropy $H_{\mathcal{S}}(\alpha) \doteq 0.6365$ nats, the logical entropy $H_{L}(\alpha) \doteq 0.444$, and the Tsallis entropies $T_{3}^{s}(\alpha) \doteq 0.333, T_{1 / 2}^{s}(\alpha) \doteq 0.7877$. We can see that it holds $T_{3}^{s}(\alpha)<H_{L}(\alpha)<T_{1 / 2}^{s}(\alpha)$, so the obtained results are consistent with the statement in the previous theorem. In addition, we have: $T_{1 / 2}^{s}(\alpha)>H_{s}(\alpha), T_{3}^{s}(\alpha)<H_{S}(\alpha), H_{L}(\alpha)<H_{s}(\alpha)$, which is consistent with what is stated in Remark 3.

In the following theorem, we show the concavity of Tsallis entropy $T_{q}^{s}(\alpha)$ as a function of $s$. Let us denote by the symbol $S(M)$ the family of all fuzzy P-measures defined on a given fuzzy measurable space $(X, M)$. It is routine to prove that if $s, t \in S(M)$, then, for every real number $\lambda \in[0,1]$, it holds that $\lambda s+(1-\lambda) t \in S(M)$.

Theorem 4. Let $\alpha$ be a fuzzy partition of fuzzy probability spaces $(X, M, s),(X, M, t)$. Then, for every real number $\lambda \in[0,1]$, the following inequality holds:

$$
\lambda T_{q}^{\mathcal{S}}(\alpha)+(1-\lambda) T_{q}^{t}(\alpha) \leq T_{q}^{\lambda s+(1-\lambda) t}(\alpha)
$$

Proof. The proof can be done using the concavity of the function $L_{q}$, in the same way as the proof of Theorem 5 in Reference [56].

As an immediate consequence of Theorem 4, we obtain the concavity of the logical entropy of the fuzzy partition on the family $S(M)$.

Theorem 5. Let $\alpha=\left\{a_{1}, a_{2}, \ldots, a_{n}\right\}$ be any fuzzy partition of $(X, M, s)$. Then $T_{q}^{s}(\alpha) \leq \frac{1}{q-1}\left(1-n^{1-q}\right)$ with the equality if and only if the fuzzy P-measure $s$ is uniform over $\alpha$, i.e., if and only if $s\left(a_{k}\right)=\frac{1}{n}$, for $k=1,2, \ldots, n$.

Proof. To prove the claim, we apply the Jensen inequality to the function $L_{q}$. Since the function $L_{q}$ is concave, setting $x_{k}=s\left(a_{k}\right)$, and $\lambda_{k}=\frac{1}{n}$, for $k=1,2, \ldots, n$, in Equation (10), we obtain:

$$
L_{q}\left(\frac{1}{n} \sum_{k=1}^{n} s\left(a_{k}\right)\right) \geq \sum_{k=1}^{n} \frac{1}{n} L_{q}\left(s\left(a_{k}\right)\right)
$$

with the equality if and only if $s\left(a_{1}\right)=s\left(a_{2}\right)=\ldots=s\left(a_{n}\right)$. Since $\sum_{k=1}^{n} s\left(a_{k}\right)=1$, it follows that:

$$
T_{q}^{s}(\alpha)=\sum_{k=1}^{n} L_{q}\left(s\left(a_{k}\right)\right) \leq n \cdot L_{q}\left(\frac{1}{n} \sum_{k=1}^{n} s\left(a_{k}\right)\right)=n \cdot L_{q}\left(\frac{1}{n}\right)=\frac{n}{q-1}\left(\frac{1}{n}-\left(\frac{1}{n}\right)^{q}\right)=\frac{1}{q-1}\left(1-n^{1-q}\right)
$$

The equality holds if and only if $s\left(a_{1}\right)=s\left(a_{2}\right)=\ldots=s\left(a_{n}\right)$, i.e., if and only if $s\left(a_{k}\right)=\frac{1}{n}$, for $k=1,2, \ldots, n$.

The following theorem shows that the Tsallis entropy $T_{q}^{s}(\alpha)$ does not satisfy the additivity property; it has the following property called pseudo-additivity.

Theorem 6. Let fuzzy partitions $\alpha, \beta$ of $(X, M, s)$ be statistically independent. Then:

$$
T_{q}^{\mathcal{S}}(\alpha \vee \beta)=T_{q}^{s}(\alpha)+T_{q}^{\mathcal{S}}(\beta)+(1-q) \cdot T_{q}^{\mathcal{S}}(\alpha) \cdot T_{q}^{\mathcal{S}}(\beta)
$$


Proof. Suppose that $\alpha=\left\{a_{1}, a_{2}, \ldots, a_{n}\right\}, \beta=\left\{b_{1}, b_{2}, \ldots, b_{m}\right\}$. We prove the equality by a direct computation:

$$
\begin{aligned}
T_{q}^{s}(\alpha \vee \beta) & =\frac{1}{q-1}\left(1-\sum_{i=1}^{n} \sum_{j=1}^{m} s\left(a_{i} \cap b_{j}\right)^{q}\right)=\frac{1}{q-1}\left(1-\sum_{i=1}^{n} s\left(a_{i}\right)^{q} \sum_{j=1}^{m} s\left(b_{j}\right)^{q}\right) \\
& =\frac{1}{q-1}\left(1-\sum_{j=1}^{m} s\left(b_{j}\right)^{q}+\sum_{j=1}^{m} s\left(b_{j}\right)^{q}-\sum_{i=1}^{n} s\left(a_{i}\right)^{q} \sum_{j=1}^{m} s\left(b_{j}\right)^{q}\right) \\
& =\frac{1}{q-1}\left(1-\sum_{j=1}^{m} s\left(b_{j}\right)^{q}\right)+\frac{1}{q-1} \sum_{j=1}^{m} s\left(b_{j}\right)^{q}\left(1-\sum_{i=1}^{n} s\left(a_{i}\right)^{q}\right) \\
& =T_{q}^{s}(\beta)+T_{q}^{s}(\alpha)+(1-q) \cdot T_{q}^{s}(\alpha) \cdot T_{q}^{s}(\beta) .
\end{aligned}
$$

Theorem 7. Let $\alpha, \beta$ be fuzzy partitions of $(X, M, s)$. Then $\alpha \prec \beta$ implies the inequality $T_{q}^{s}(\alpha) \leq T_{q}^{s}(\beta)$.

Proof. Let us assume that $\alpha=\left\{a_{1}, a_{2}, \ldots, a_{n}\right\}, \beta=\left\{b_{1}, b_{2}, \ldots, b_{m}\right\}, \alpha \prec \beta$. Then there exists a partition $\left\{I_{1}, I_{2}, \ldots, I_{n}\right\}$ of the set $\{1,2, \ldots, m\}$ such that $a_{i}=\cup_{j \in I_{i}} b_{j}$, for $i=1,2, \ldots, n$. Therefore, we have $s\left(a_{i}\right)=s\left(\cup_{j \in I_{i}} b_{j}\right)=\sum_{j \in I_{i}} s\left(b_{j}\right)$, for $i=1,2, \ldots, n$. Let us consider the case of $q \in(0,1)$. Then we have:

$$
s\left(a_{i}\right)^{q}=\left(\sum_{j \in I_{i}} s\left(b_{j}\right)\right)^{q} \leq \sum_{j \in I_{i}} s\left(b_{j}\right)^{q}
$$

for $i=1,2, \ldots, n$. If we add these inequalities with respect to $i=1,2, \ldots, n$, then we obtain:

$$
\sum_{i=1}^{n} s\left(a_{i}\right)^{q} \leq \sum_{i=1}^{n} \sum_{j \in I_{i}} s\left(b_{j}\right)^{q}=\sum_{j=1}^{m} s\left(b_{j}\right)^{q} .
$$

Since in this case, it holds that $\frac{1}{q-1}<0$, it follows that:

$$
T_{q}^{s}(\alpha)=\frac{1}{q-1}\left(1-\sum_{i=1}^{n} s\left(a_{i}\right)^{q}\right) \leq \frac{1}{q-1}\left(1-\sum_{j=1}^{m} s\left(b_{j}\right)^{q}\right)=T_{q}^{s}(\beta) .
$$

The case of $q \in(1, \infty)$ can be proven in an analogous way.

Next, we will need the following proposition.

Proposition 3. Let $\alpha=\left\{a_{1}, a_{2}, \ldots, a_{n}\right\}, \beta=\left\{b_{1}, b_{2}, \ldots, b_{m}\right\}$ be fuzzy partitions of $(X, M, s)$, and $q>1$. Then:

$$
\sum_{j=1}^{m} s\left(b_{j}\right)^{q} \sum_{i=1}^{n} L_{q}\left(s\left(a_{i} / b_{j}\right)\right) \leq T_{q}^{s}(\alpha)
$$

Proof. We assume that $q>1$; therefore, for $j=1,2, \ldots, m$, it holds that $s\left(b_{j}\right)^{q} \leq s\left(b_{j}\right)$. Since the function $L_{q}$ is non-negative, it follows that:

$$
s\left(b_{j}\right)^{q} \sum_{i=1}^{n} L_{q}\left(s\left(a_{i} / b_{j}\right)\right) \leq s\left(b_{j}\right) \sum_{i=1}^{n} L_{q}\left(s\left(a_{i} / b_{j}\right)\right),
$$

for $j=1,2, \ldots, m$. If we add these inequalities with respect to $j=1,2, \ldots, m$, we obtain:

$$
\sum_{j=1}^{m} s\left(b_{j}\right)^{q} \sum_{i=1}^{n} L_{q}\left(s\left(a_{i} / b_{j}\right)\right) \leq \sum_{j=1}^{m} s\left(b_{j}\right) \sum_{i=1}^{n} L_{q}\left(s\left(a_{i} / b_{j}\right)\right)
$$


Next, we apply the Jensen inequality to the concave function $L_{q}$. If we insert into Equation (10) $x_{j}=s\left(a_{i} / b_{j}\right)$, and $\lambda_{j}=s\left(b_{j}\right)$, for $j=1,2, \ldots, m$, then we obtain:

$$
\sum_{j=1}^{m} s\left(b_{j}\right) L_{q}\left(s\left(a_{i} / b_{j}\right)\right) \leq L_{q}\left(\sum_{j=1}^{m} s\left(b_{j}\right) s\left(a_{i} / b_{j}\right)\right)=L_{q}\left(\sum_{j=1}^{m} s\left(a_{i} \cap b_{j}\right)\right)=L_{q}\left(s\left(a_{i}\right)\right),
$$

for $i=1,2, \ldots, n$. It follows that:

$$
\sum_{j=1}^{m} s\left(b_{j}\right) \sum_{i=1}^{n} L_{q}\left(s\left(a_{i} / b_{j}\right)\right) \leq \sum_{i=1}^{n} L_{q}\left(s\left(a_{i}\right)\right)=T_{q}^{s}(\alpha) .
$$

By combining the previous results, we obtain the assertion.

In the following theorem, it is stated that the Tsallis entropy of fuzzy partitions of order $q>1$ satisfies the sub-additivity property. As shown in the example that follows, the Tsallis entropy of order $q \in(0,1)$ does not satisfy the property of sub-additivity in general.

Theorem 8. Let $\alpha, \beta$ be fuzzy partitions of $(X, M, s)$, and $q>1$. Then $T_{q}^{s}(\alpha \vee \beta) \leq T_{q}^{s}(\alpha)+T_{q}^{s}(\beta)$.

Proof. The proof can be done using part (i) of Proposition 1 and Proposition 3, in the same way as the proof of Theorem 3 in Reference [56].

Example 4. Consider any fuzzy probability space $(X, M, s)$, and two fuzzy events $a, b$ of $M$ with $s(a)=0.5$, $s(b)=0.4$. Then the families $\alpha=\left\{a, a^{\perp}\right\}, \beta=\left\{b, b^{\perp}\right\}$ are fuzzy partitions of $(X, M, s)$ with the s-values $0.5,0.5$ and $0.4,0.6$ of the corresponding elements, respectively. Elementary calculations show that the fuzzy partition $\alpha$ has the Shannon entropy $H_{S}(\alpha) \doteq 0.6931$ nats, the logical entropy $H_{L}(\alpha)=0.5$, and the Tsallis entropies $T_{4}^{s}(\alpha) \doteq 0.292, T_{1 / 3}^{s}(\alpha) \doteq 0.8811$; the fuzzy partition $\beta$ has the Shannon entropy $H_{s}(\beta) \doteq 0.673$ nats, the logical entropy $H_{L}(\beta)=0.48$, and the Tsallis entropies $T_{4}^{s}(\beta) \doteq 0.2816, T_{1 / 3}^{s}(\beta) \doteq 0.8704$. Let us assume that the fuzzy partitions $\alpha$ and $\beta$ are statistically independent. Then the fuzzy partition $\alpha \vee \beta=$ $\left\{a \cap b, a \cap b^{\perp}, a^{\perp} \cap b, a^{\perp} \cap b^{\perp}\right\}$ has the s-values $0.2,0.3,0.2,0.3$ of the corresponding elements. Elementary calculations show that $H_{s}(\alpha \vee \beta) \doteq 1.366$ nats, $H_{L}(\alpha \vee \beta)=0.74, T_{4}^{s}(\alpha \vee \beta) \doteq 0.327$, and $T_{1 / 3}^{s}(\alpha \vee \beta) \doteq$ 2.263. It can be seen that $H_{s}(\alpha \vee \beta)=H_{s}(\alpha)+H_{s}(\beta), H_{L}(\alpha \vee \beta)<H_{L}(\alpha)+H_{L}(\beta)$, and $T_{4}^{s}(\alpha \vee \beta)<$ $T_{4}^{s}(\alpha)+T_{4}^{s}(\beta)$. On the other hand it holds that $T_{1 / 3}^{s}(\alpha \vee \beta)>T_{1 / 3}^{s}(\alpha)+T_{1 / 3}^{s}(\beta)$. This means that the Tsallis entropy $T_{q}^{s}(\alpha)$ of order $q \in(0,1)$ does not satisfy the sub-additivity property in general.

Theorem 9. Let $\alpha, \beta$, $\gamma$ be fuzzy partitions of $(X, M, s)$. Then:

(i) $T_{q}^{s}(\alpha / \beta) \geq 0$;

(ii) $T_{q}^{s}(\alpha \vee \beta / \gamma)=T_{q}^{s}(\alpha / \gamma)+T_{q}^{s}(\beta / \alpha \vee \gamma)$;

(iii) $T_{q}^{s}(\alpha \vee \beta)=T_{q}^{s}(\alpha)+T_{q}^{s}(\beta / \alpha)$.

Proof. Let $\alpha=\left\{a_{1}, a_{2}, \ldots, a_{l}\right\}, \beta=\left\{b_{1}, b_{2}, \ldots, b_{m}\right\}, \gamma=\left\{c_{1}, c_{2}, \ldots, c_{n}\right\}$.

(i) According to Proposition 1 , we have $s\left(b_{j}\right)=\sum_{i=1}^{l} s\left(a_{i} \cap b_{j}\right)$, for $j=1,2, \ldots, m$. Therefore, we obtain:

$$
\begin{gathered}
T_{q}^{s}(\alpha / \beta)=\frac{1}{q-1}\left(\sum_{j=1}^{m} s\left(b_{j}\right)^{q}-\sum_{i=1}^{l} \sum_{j=1}^{m} s\left(a_{i} \cap b_{j}\right)^{q}\right)=\frac{1}{q-1}\left(\sum_{j=1}^{m} s\left(b_{j}\right)^{q-1} \sum_{i=1}^{l} s\left(a_{i} \cap b_{j}\right)-\sum_{i=1}^{l} \sum_{j=1}^{m} s\left(a_{i} \cap b_{j}\right)^{q}\right) \\
=\frac{1}{q-1} \sum_{i=1}^{l} \sum_{j=1}^{m} s\left(a_{i} \cap b_{j}\right)\left(s\left(b_{j}\right)^{q-1}-s\left(a_{i} \cap b_{j}\right)^{q-1}\right) .
\end{gathered}
$$

Consider the case of $q \in(0,1)$. For $i=1,2, \ldots, l, j=1,2, \ldots, m$, we have $s\left(a_{i} \cap b_{j}\right) \leq s\left(b_{j}\right)$, which implies that $s\left(a_{i} \cap b_{j}\right)^{q-1} \geq s\left(b_{j}\right)^{q-1}$, for $i=1,2, \ldots, l, j=1,2, \ldots, m$. Since $\frac{1}{q-1}<0$, for $q \in(0,1)$, 
it follows that $T_{q}^{s}(\alpha / \beta) \geq 0$. On the other hand, for $q \in(1, \infty)$, we have $s\left(a_{i} \cap b_{j}\right)^{q-1} \leq s\left(b_{j}\right)^{q-1}$, for $i=1,2, \ldots, l, j=1,2, \ldots, m$. In this case, $\frac{1}{q-1}>0$, hence $T_{q}^{s}(\alpha / \beta) \geq 0$.

(ii) Let us calculate:

$$
\begin{aligned}
& T_{q}^{s}(\alpha / \gamma)+T_{q}^{s}(\beta / \alpha \vee \gamma)=\frac{1}{q-1}\left(\sum_{k=1}^{n} s\left(c_{k}\right)^{q}-\sum_{i=1}^{l} \sum_{k=1}^{n} s\left(a_{i} \cap c_{k}\right)^{q}\right) \\
& +\frac{1}{q-1}\left(\sum_{i=1}^{l} \sum_{k=1}^{n} s\left(a_{i} \cap c_{k}\right)^{q}-\sum_{i=1}^{l} \sum_{j=1}^{m} \sum_{k=1}^{n} s\left(a_{i} \cap b_{j} \cap c_{k}\right)^{q}\right) \\
& =\frac{1}{q-1}\left(\sum_{k=1}^{n} s\left(c_{k}\right)^{q}-\sum_{i=1}^{l} \sum_{j=1}^{m} \sum_{k=1}^{n} s\left(a_{i} \cap b_{j} \cap c_{k}\right)^{q}\right)=T_{q}^{s}(\alpha \vee \beta / \gamma) .
\end{aligned}
$$

(iii) It is sufficient to set $\gamma=\left\{1_{X}\right\}$ in (ii).

As a direct consequence of the claims (i) and (iii) of the previous theorem, the following property of Tsallis entropy of fuzzy partitions is obtained.

Theorem 10. For arbitrary fuzzy partitions $\alpha, \beta$ of $(X, M, s)$, the following inequality holds:

$$
T_{q}^{S}(\alpha \vee \beta) \geq \max \left[T_{q}^{S}(\alpha), T_{q}^{S}(\beta)\right]
$$

Theorem 11. (Chain rules for Tsallis entropy). Let $\alpha_{1}, \alpha_{2}, \ldots, \alpha_{n}$ and $\beta$ be fuzzy partitions of $(X, M, s)$. Put $\alpha_{0}=\left\{1_{X}\right\}$. Then the following equalities hold:

(i) $T_{q}^{s}\left(\vee_{i=1}^{n} \alpha_{i} / \beta\right)=\sum_{i=1}^{n} T_{q}^{s}\left(\alpha_{i} /\left(\vee_{k=0}^{i-1} \alpha_{k}\right) \vee \beta\right)$;

(ii) $T_{q}^{s}\left(\vee_{i=1}^{n} \alpha_{i}\right)=\sum_{i=1}^{n} T_{q}^{s}\left(\alpha_{i} / \vee_{k=0}^{i-1} \alpha_{k}\right)$.

Proof. The proof of claim (i) can be made using mathematical induction and the property (ii) of Theorem 9. If we put $\beta=\left\{1_{X}\right\}$ in claim (i), then equality (ii) is obtained.

Theorem 12. Let fuzzy partitions $\alpha, \beta$ of $(X, M, s)$ be statistically independent. Then:

$$
T_{q}^{S}(\alpha / \beta)=T_{q}^{s}(\alpha)+(1-q) \cdot T_{q}^{s}(\alpha) \cdot T_{q}^{s}(\beta) .
$$

Proof. The claim is obtained by combining property (iii) from Theorem 9 with Theorem 6 .

Theorem 13. Let $\alpha, \beta$ be fuzzy partitions of $(X, M, s)$. Then, for $q>1$, it holds that:

$$
T_{q}^{s}(\alpha / \beta) \leq T_{q}^{s}(\alpha)
$$

Proof. The claim is obtained by combining property (iii) from Theorem 9 with Theorem 8 .

The following example, which is a continuation of Example 4, illustrates the result of Theorem 13 and shows that the conditional Tsallis entropy $T_{q}^{s}(\alpha / \beta)$ of order $q \in(0,1)$ does not have the monotonicity property in general.

Example 5. Let us consider the fuzzy probability space $(X, M, s)$, and the partitions $\alpha, \beta$ from the previous example. We computed the Tsallis entropies to be $T_{4}^{s}(\alpha) \doteq 0.292, T_{4}^{s}(\beta) \doteq 0.2816, T_{1 / 3}^{s}(\alpha) \doteq 0.8811$, and $T_{1 / 3}^{s}(\beta) \doteq 0.8704$. Through easy calculations, we find that $T_{4}^{s}(\alpha / \beta) \doteq 0.0453, T_{1 / 3}^{s}(\alpha / \beta) \doteq 1.392$, $T_{4}^{s}(\beta / \alpha) \doteq 0.0352$, and $T_{1 / 3}^{s}(\beta / \alpha) \doteq 1.3816$. Evidently, we have $T_{4}^{s}(\alpha / \beta)<T_{4}^{s}(\alpha)$, and $T_{4}^{s}(\beta / \alpha)<T_{4}^{s}(\beta)$, which is consistent with the assertion of Theorem 13. On the other hand, we have $T_{1 / 3}^{s}(\alpha / \beta)>T_{1 / 3}^{s}(\alpha)$, and $T_{1 / 3}^{s}(\beta / \alpha)>T_{1 / 3}^{s}(\beta)$. That is, the conditional Tsallis entropy $T_{q}^{s}(\alpha / \beta)$ of order $q \in(0,1)$ does not have the monotonicity property in general. 


\section{Tsallis Entropy of Fuzzy Dynamical Systems}

In this section, we introduce and study the concept of the Tsallis entropy of a fuzzy dynamical system.

Definition 9 [29]. By a fuzzy dynamical system, we understand a quadruple $(X, M, s, \tau)$, where $(X, M, s)$ is a fuzzy probability space and $\tau: M \rightarrow M$ is a mapping with the following properties: (i) $s(a)=s(\tau(a))$, for every $a \in M$; (ii) $\tau\left(\cup_{n=1}^{\infty} a_{n}\right)=\cup_{n=1}^{\infty} \tau\left(a_{n}\right)$, for any sequence $\left\{a_{n}\right\}_{n=1}^{\infty} \subset M$; (iii) $\tau\left(a^{\perp}\right)=(\tau(a))^{\perp}$; for every $a \in M$.

Example 6. Let $(X, M, s)$ be a fuzzy probability space and let $U: X \rightarrow X$ be such a transformation that the following two conditions are satisfied: $a \in M$ implies $a \circ U \in M$, and $s(a \circ U)=s(a)$, for every $a \in M$. If we define the mapping $\tau: M \rightarrow M$ by the following equation:

$$
\tau(a)=a \circ U,
$$

for every $a \in M$, then it is possible to verify that the system $(X, M, s, \tau)$ is a fuzzy dynamical system.

Example 7. A classical dynamical system $(X, S, \mu, U)$ can be viewed as a fuzzy dynamical system $(X, M, s, \tau)$, if we consider the fuzzy probability space $(X, M, s)$ from Example 1, and define the mapping $\tau: M \rightarrow M$ by $\tau\left(\varphi_{A}\right)=\varphi_{A} \circ U=\varphi_{U^{-1}(A)}$, for every $\varphi_{A} \in M$. In this way, the classical model can be inserted into the fuzzy case.

Let $(X, M, s, \tau)$ be a fuzzy dynamical system and $\alpha=\left\{a_{1}, a_{2}, \ldots, a_{n}\right\}$ be a fuzzy partition of $(X, M, s)$. It can be verified that the family $\tau(\alpha)=\left\{\tau\left(a_{1}\right), \tau\left(a_{2}\right), \ldots, \tau\left(a_{n}\right)\right\}$ is a fuzzy partition of $(X, M, s)$. Indeed, we have $s\left(\cup_{i=1}^{n} \tau\left(a_{i}\right)\right)=s\left(\tau\left(\cup_{i=1}^{n} a_{i}\right)\right)=s\left(\cup_{i=1}^{n} a_{i}\right)=1$, and $\tau\left(a_{i}\right) \cap\left(\tau\left(a_{j}\right)\right)^{\perp}=$ $\tau\left(a_{i} \cap a_{j}{ }^{\perp}\right)=\tau\left(a_{i}\right)$, whenever $i \neq j$. Let $\alpha, \beta$ be two fuzzy partitions of $(X, M, s)$. Then, in view of Definition 9, we have the equality $\tau(\alpha \vee \beta)=\tau(\alpha) \vee \tau(\beta)$. Further, define $\tau^{k}: M \rightarrow M$, for $k=$ $0,1,2, \ldots$, by induction on $k$, by setting $\tau^{k+1}=\tau \circ \tau^{k}$, where $\tau^{0}: M \rightarrow M$ is the identical map. Evidently, the map $\tau^{k}: M \rightarrow M$ satisfies properties (i)-(iii) of the definition above. This means that, for any non-negative integer $k$, the system $\left(X, M, s, \tau^{k}\right)$ is a fuzzy dynamical system.

Proposition 4. Let $(X, M, s, \tau)$ be a fuzzy dynamical system and let $\alpha, \beta$ be fuzzy partitions of $(X, M, s)$ such that $\alpha \prec \beta$. Then $\tau(\alpha) \prec \tau(\beta)$.

Proof. Suppose that $\alpha=\left\{a_{1}, a_{2}, \ldots, a_{n}\right\}, \beta=\left\{b_{1}, b_{2}, \ldots, b_{m}\right\}, \alpha \prec \beta$. Then there exists a partition $\left\{I_{1}, I_{2}, \ldots, I_{n}\right\}$ of the set $\{1,2, \ldots, m\}$ such that $a_{i}=\cup_{j \in I_{i}} b_{j}$, for $i=1,2, \ldots, n$. Therefore, by condition (ii) from Definition 9, we obtain $\tau\left(a_{i}\right)=\tau\left(\cup_{j \in I_{i}} B_{j}\right)=\cup_{j \in I_{i}} \tau\left(B_{j}\right)$, for $i=1,2, \ldots, n$. It follows that $\tau(\alpha) \prec \tau(\beta)$.

Theorem 14. Let $(X, M, s, \tau)$ be a fuzzy dynamical system and let $\alpha, \beta$ be fuzzy partitions of $(X, M, s)$. Then:

(i) $T_{q}^{s}\left(\tau^{n}(\alpha)\right)=T_{q}^{s}(\alpha)$, for $n=0,1,2, \ldots$;

(ii) $T_{q}^{s}\left(\tau^{n}(\alpha) / \tau^{n}(\beta)\right)=T_{q}^{s}(\alpha / \beta)$, for $n=0,1,2, \ldots$;

(iii) $T_{q}^{s}\left(\vee_{k=0}^{n-1} \tau^{k}(\alpha)\right)=T_{q}^{s}(\alpha)+\sum_{i=1}^{n-1} T_{q}^{s}\left(\alpha / \vee_{k=1}^{i} \tau^{k}(\alpha)\right)$, for $n=2,3, \ldots$

Proof. Properties (i) and (ii) are obvious consequences of condition (i) from Definition 9. By using property (iii) of Theorem 9 and mathematical induction on $n$ starting with $n=2$, we obtain the equality (iii).

The aim of this section is to define the Tsallis entropy of order $q>1$ of a fuzzy dynamical system. First, we prove the following proposition, which plays a key role. 
Proposition 5. Let $(X, M, s, \tau)$ be a fuzzy dynamical system and let $\alpha$ be a fuzzy partition of $(X, M, s)$. Then, for $q>1$, the following limit exists:

$$
\lim _{n \rightarrow \infty} \frac{1}{n} T_{q}^{S}\left(\vee_{k=0}^{n-1} \tau^{k}(\alpha)\right)
$$

Proof. In order to prove the claim, we use Theorem 4.9 of Reference [62], which says that if $\left\{c_{n}\right\}_{n=1}^{\infty}$ is a sub-additive sequence of non-negative real numbers (i.e., $c_{n} \geq 0$, and $c_{n+m} \leq c_{n}+c_{m}$, for every natural number $n, m)$, then $\lim _{n \rightarrow \infty} \frac{1}{n} c_{n}$ exists. Let us denote by $c_{n}$ the number $T_{q}^{s}\left(\vee_{k=0}^{n-1} \tau^{k}(\alpha)\right), n=1,2, \ldots$ The Tsallis entropy is always nonnegative; thus, $c_{n} \geq 0$, for $n=1,2, \ldots$ In addition, according to the sub-additivity property of Tsallis entropy $T_{q}^{s}(\alpha)$ of order $q>1$, and property (i) from Theorem 14, we obtain:

$$
\begin{gathered}
c_{n+m}=T_{q}^{s}\left(\bigvee_{k=0}^{n+m-1} \tau^{k}(\alpha)\right) \leq T_{q}^{s}\left(\vee_{k=0}^{n-1} \tau^{k}(\alpha)\right)+T_{q}^{s}\left(\bigvee_{k=n}^{n+m-1} \tau^{k}(\alpha)\right) \\
=c_{n}+T_{q}^{s}\left(\tau^{n}\left(\vee_{k=0}^{m-1} \tau^{k}(\alpha)\right)\right)=c_{n}+T_{q}^{s}\left(\vee_{k=0}^{m-1} \tau^{k}(\alpha)\right)=c_{n}+c_{m} .
\end{gathered}
$$

Therefore, $\lim _{n \rightarrow \infty} \frac{1}{n} c_{n}$ exists.

Definition 10. Let $(X, M, s, \tau)$ be a fuzzy dynamical system and let $\alpha$ be a fuzzy partition of $(X, M, s)$. Then, for $q>1$, we define the Tsallis entropy of $\tau$ with respect to $\alpha$ by:

$$
T_{q}^{s}(\tau, \alpha)=\lim _{n \rightarrow \infty} \frac{1}{n} T_{q}^{s}\left(\vee_{k=0}^{n-1} \tau^{k}(\alpha)\right) .
$$

Theorem 15. Let $(X, M, s, \tau)$ be a fuzzy dynamical system and let $\alpha$ be a fuzzy partition of $(X, M, s)$. Then, for $q>1$, and for any non-negative integer $k$, the following equality holds:

$$
T_{q}^{s}(\tau, \alpha)=T_{q}^{s}\left(\tau, \vee_{i=0}^{k} \tau^{i}(\alpha)\right)
$$

Proof. In view of Definition 10, we can write:

$$
\begin{aligned}
& T_{q}^{s}\left(\tau, \vee_{i=0}^{k} \tau^{i}(\alpha)\right)=\lim _{n \rightarrow \infty} \frac{1}{n} T_{q}^{s}\left(\vee_{j=0}^{n-1} \tau^{j}\left(\bigvee_{i=0}^{k} \tau^{i}(\alpha)\right)\right) \\
& =\lim _{n \rightarrow \infty} \frac{k+n}{n} \cdot \frac{1}{k+n} T_{q}^{s}\left(\vee_{i=0}^{k+n-1} \tau^{i}(\alpha)\right) \\
& =\lim _{n \rightarrow \infty} \frac{1}{k+n} T_{q}^{s}\left(\vee_{i=0}^{k+n-1} \tau^{i}(\alpha)\right)=T_{q}^{s}(\tau, \alpha) .
\end{aligned}
$$

Theorem 16. Let $(X, M, s, \tau)$ be a fuzzy dynamical system and let $\alpha, \beta$ be fuzzy partitions of $(X, M, s)$. Then, for $q>1, \alpha \prec \beta$ implies $T_{q}^{s}(\tau, \alpha) \leq T_{q}^{s}(\tau, \beta)$.

Proof. Let $\alpha, \beta$ be fuzzy partitions of $(X, M, s)$ such that $\alpha \prec \beta$. Then, by combining Proposition 2 with Proposition 4 and using mathematical induction, we find that $\vee_{k=0}^{n-1} \tau^{k}(\alpha) \prec \vee_{k=0}^{n-1} \tau^{k}(\beta)$, for $n=1,2, \ldots$ Hence, according to Theorem 7, we have:

$$
T_{q}^{s}\left(\vee_{k=0}^{n-1} \tau^{k}(\alpha)\right) \leq T_{q}^{s}\left(\vee_{k=0}^{n-1} \tau^{k}(\beta)\right)
$$

for $n=1,2, \ldots$ It follows that:

$$
T_{q}^{s}(\tau, \alpha)=\lim _{n \rightarrow \infty} \frac{1}{n} T_{q}^{s}\left(\vee_{k=0}^{n-1} \tau^{k}(\alpha)\right) \leq \lim _{n \rightarrow \infty} \frac{1}{n} T_{q}^{s}\left(\vee_{k=0}^{n-1} \tau^{k}(\beta)\right)=T_{q}^{s}(\tau, \beta) .
$$

Definition 11. The Tsallis entropy of a fuzzy dynamical system $(X, M, s, \tau)$ is defined, for $q>1$, by the following equation:

$$
T_{q}^{\mathcal{S}}(\tau)=\sup \left\{T_{q}^{\mathcal{S}}(\tau, \alpha)\right\},
$$


where the supremum is taken over all fuzzy partitions $\alpha$ of $(X, M, s)$.

Example 8. A trivial case of a fuzzy dynamical system is a system $(X, M, s, I)$, where $(X, M, s)$ is a fuzzy probability space and $I: M \rightarrow M$ is the identity map. The operation $\vee$ is idempotent; therefore, for every fuzzy partition $\alpha$ of $(X, M, s)$, we have:

$$
T_{q}^{S}(I, \alpha)=\lim _{n \rightarrow \infty} \frac{1}{n} T_{q}^{S}\left(\vee_{k=0}^{n-1} I^{k}(\alpha)\right)=\lim _{n \rightarrow \infty} \frac{1}{n} T_{q}^{S}(\alpha)=0
$$

The Tsallis entropy of the fuzzy dynamical system $(X, M, s, I)$ is $T_{q}^{s}(I)=\sup \left\{T_{q}^{s}(I, \alpha) ; \alpha\right.$ is a fuzzy partition of $(X, M, s)\}=0$.

Definition 12. Two fuzzy dynamical systems $\left(X_{1}, M_{1}, s_{1}, \tau_{1}\right),\left(X_{2}, M_{2}, s_{2}, \tau_{2}\right)$ are called isomorphic if there exists a bijective map $\Psi: M_{1} \rightarrow M_{2}$ such that the following conditions are satisfied:

(i) $\Psi$ preserves the operations, i.e., $\Psi\left(\cup_{n=1}^{\infty} a_{n}\right)=\cup_{n=1}^{\infty} \Psi\left(a_{n}\right)$, for any sequence $\left\{a_{n}\right\}_{n=1}^{\infty} \subset M_{1}$, and $\Psi\left(a^{\perp}\right)=(\Psi(a))^{\perp}$, for every $a \in M_{1}$;

(ii) $s_{2}(\Psi(a))=s_{1}(a)$, for every $a \in M_{1}$;

(iii) $\Psi\left(\tau_{1}(a)\right)=\tau_{2}(\Psi(a))$, for every $a \in M_{1}$.

The map $\Psi$ is called an isomorphism.

Proposition 6. Let $\left(X_{1}, M_{1}, s_{1}, \tau_{1}\right),\left(X_{2}, M_{2}, s_{2}, \tau_{2}\right)$ be isomorphic fuzzy dynamical systems and $\Psi: M_{1} \rightarrow M_{2}$ be an isomorphism between them. Let $\alpha=\left\{a_{1}, a_{2}, \ldots, a_{n}\right\}$ be a fuzzy partition of $\left(X_{1}, M_{1}, s_{1}\right)$. Then the family $\Psi(\alpha)=\left\{\Psi\left(a_{1}\right), \Psi\left(a_{2}\right), \ldots, \Psi\left(a_{n}\right)\right\}$ is a fuzzy partition of $\left(X_{2}, M_{2}, s_{2}\right)$ with the Tsallis entropy $T_{q}^{s_{2}}(\Psi(\alpha))=T_{q}^{s_{1}}(\alpha)$. In addition, for $q>1$, it holds that $T_{q}^{s_{2}}\left(\tau_{2}, \Psi(\alpha)\right)=T_{q}^{s_{1}}\left(\tau_{1}, \alpha\right)$.

Proof. By the above assumption, we obtain:

$$
s_{2}\left(\cup_{i=1}^{n} \Psi\left(a_{i}\right)\right)=s_{2}\left(\Psi\left(\cup_{i=1}^{n} a_{i}\right)\right)=s_{1}\left(\cup_{i=1}^{n} a_{i}\right)=1 \text {, }
$$

and

$$
\Psi\left(a_{i}\right) \cap\left(\Psi\left(a_{j}\right)\right)^{\perp}=\Psi\left(a_{i}\right) \cap \Psi\left(a_{j}^{\perp}\right)=\Psi\left(a_{i} \cap a_{j}^{\perp}\right)=\Psi\left(a_{i}\right),
$$

whenever $i \neq j$. Thus, $\Psi(\alpha)=\left\{\Psi\left(a_{1}\right), \Psi\left(a_{2}\right), \ldots, \Psi\left(a_{n}\right)\right\}$ is a fuzzy partition of $\left(X_{2}, M_{2}, s_{2}\right)$. Let us calculate its Tsallis entropy:

$$
T_{q}^{s_{2}}(\Psi(\alpha))=\sum_{i=1}^{n} L_{q}\left(s_{2}\left(\Psi\left(a_{i}\right)\right)\right)=\sum_{i=1}^{n} L_{q}\left(s_{1}\left(a_{i}\right)\right)=T_{q}^{s_{1}}(\alpha) .
$$

We used Equation (13) and condition (ii) of Definition 12. Consequently, by the use of conditions (iii) and (i) from Definition 12, for $q>1$, we obtain:

$$
\begin{gathered}
T_{q}^{\mathcal{S}_{2}}\left(\tau_{2}, \Psi(\alpha)\right)=\lim _{n \rightarrow \infty} \frac{1}{n} T_{q}^{\mathcal{S}_{2}}\left(\bigvee_{k=0}^{n-1} \tau_{2}{ }^{k}(\Psi(\alpha))\right)=\lim _{n \rightarrow \infty} \frac{1}{n} T_{q}^{\mathcal{S}_{2}}\left(\mathrm{\vee}_{k=0}^{n-1} \Psi\left(\tau_{1}{ }^{k}(\alpha)\right)\right) \\
=\lim _{n \rightarrow \infty} \frac{1}{n} T_{q}^{\mathcal{S}_{2}}\left(\Psi\left(\bigvee_{k=0}^{n-1} \tau_{1}{ }^{k}(\alpha)\right)\right)=\lim _{n \rightarrow \infty} \frac{1}{n} T_{q}^{\mathcal{S}_{1}}\left(\mathrm{~V}_{k=0}^{n-1} \tau_{1}{ }^{k}(\alpha)\right)=T_{q}^{\mathcal{S}_{1}}\left(\tau_{1}, \alpha\right) .
\end{gathered}
$$

Proposition 7. Let $\left(X_{1}, M_{1}, s_{1}, \tau_{1}\right),\left(X_{2}, M_{2}, s_{2}, \tau_{2}\right)$ be isomorphic fuzzy dynamical systems and $\Psi: M_{1} \rightarrow M_{2}$ be an isomorphism between them. Then, for the inverse $\Psi^{-1}: M_{2} \rightarrow M_{1}$, the following properties are satisfied:

(i) $\Psi^{-1}\left(\cup_{n=1}^{\infty} a_{n}\right)=\cup_{n=1}^{\infty} \Psi^{-1}\left(a_{n}\right)$, for any sequence $\left\{a_{n}\right\}_{n=1}^{\infty} \subset M_{2}$;

(ii) $\Psi^{-1}\left(a^{\perp}\right)=\left(\Psi^{-1}(a)\right)^{\perp}$, for every $a \in M_{2}$; 
(iii) $s_{1}\left(\Psi^{-1}(a)\right)=s_{2}(a)$, for every $a \in M_{2}$;

(iv) $\Psi^{-1}\left(\tau_{2}(a)\right)=\tau_{1}\left(\Psi^{-1}(a)\right)$, for every $a \in M_{2}$.

Proof. Let $\left\{a_{n}\right\}_{n=1}^{\infty}$ be a sequence of fuzzy sets from $M_{2}$, and $a \in M_{2}$. The map $\Psi: M_{1} \rightarrow M_{2}$ is bijective; hence, there exists a sequence $\left\{a_{n}^{\prime}\right\}_{n=1}^{\infty} \subset M_{1}$ such that $\Psi^{-1}\left(a_{n}\right)=a_{n}^{\prime}$, for $n=1,2, \ldots$, and there exists $a^{\prime} \in M_{1}$ such that $\Psi^{-1}(a)=a^{\prime}$. Therefore, we obtain:

(i) $\quad \Psi^{-1}\left(\cup_{n=1}^{\infty} a_{n}\right)=\Psi^{-1}\left(\cup_{n=1}^{\infty} \Psi\left(a^{\prime}{ }_{n}\right)\right)=\Psi^{-1}\left(\Psi\left(\cup_{n=1}^{\infty} a_{n}{ }_{n}\right)\right)=\cup_{n=1}^{\infty} a^{\prime}{ }_{n}=\cup_{n=1}^{\infty} \Psi^{-1}\left(a_{n}\right)$;

(ii) $\Psi^{-1}\left(a^{\perp}\right)=\Psi^{-1}\left(\left(\Psi\left(a^{\prime}\right)\right)^{\perp}\right)=\Psi^{-1}\left(\Psi\left(\left(a^{\prime}\right)^{\perp}\right)\right)=\left(a^{\prime}\right)^{\perp}=\left(\Psi^{-1}(a)\right)^{\perp}$;

(iii) $s_{2}(a)=s_{2}\left(\Psi\left(a^{\prime}\right)\right)=s_{1}\left(a^{\prime}\right)=s_{1}\left(\Psi^{-1}(a)\right)$;

(iv) $\Psi^{-1}\left(\tau_{2}(a)\right)=\Psi^{-1}\left(\tau_{2}\left(\Psi\left(a^{\prime}\right)\right)\right)=\Psi^{-1}\left(\Psi\left(\tau_{1}\left(a^{\prime}\right)\right)\right)=\tau_{1}\left(a^{\prime}\right)=\tau_{1}\left(\Psi^{-1}(a)\right)$.

Theorem 17. Let $\left(X_{1}, M_{1}, s_{1}, \tau_{1}\right),\left(X_{2}, M_{2}, s_{2}, \tau_{2}\right)$ be isomorphic fuzzy dynamical systems and let $q>1$. Then:

$$
T_{q}^{s_{1}}\left(\tau_{1}\right)=T_{q}^{s_{2}}\left(\tau_{2}\right)
$$

Proof. Let $\alpha=\left\{a_{1}, a_{2}, \ldots, a_{n}\right\}$ be a fuzzy partition of $\left(X_{1}, M_{1}, s_{1}\right)$, and $\Psi: M_{1} \rightarrow M_{2}$ be an isomorphism between fuzzy dynamical systems $\left(X_{1}, M_{1}, s_{1}, \tau_{1}\right),\left(X_{2}, M_{2}, s_{2}, \tau_{2}\right)$. Then, according to Proposition 6, the family $\Psi(\alpha)=\left\{\Psi\left(a_{1}\right), \Psi\left(a_{2}\right), \ldots, \Psi\left(a_{n}\right)\right\}$ is a fuzzy partition of $\left(X_{2}, M_{2}, s_{2}\right)$ and it holds that $T_{q}^{s_{1}}\left(\tau_{1}, \alpha\right)=T_{q}^{s_{2}}\left(\tau_{2}, \Psi(\alpha)\right)$. It follows that:

$$
\begin{aligned}
& \left\{T_{q}^{s_{1}}\left(\tau_{1}, \alpha\right) ; \alpha \text { is a fuzzy partition of }\left(X_{1}, M_{1}, s_{1}\right)\right\} \\
\subset & \left\{T_{q}^{s_{2}}\left(\tau_{2}, \beta\right) ; \beta \text { is a fuzzy partition of }\left(X_{2}, M_{2}, s_{2}\right)\right\},
\end{aligned}
$$

and consequently:

$$
T_{q}^{s_{1}}\left(\tau_{1}\right)=\sup \left\{T_{q}^{s_{1}}\left(\tau_{1}, \alpha\right)\right\} \leq \sup \left\{T_{q}^{s_{2}}\left(\tau_{2}, \beta\right)\right\}=T_{q}^{s_{2}}\left(\tau_{2}\right),
$$

where the supremum on the left side of the inequality is taken over all fuzzy partitions $\alpha$ of $\left(X_{1}, M_{1}, s_{1}\right)$, and the supremum on the right side of the inequality is taken over all fuzzy partitions $\beta$ of $\left(X_{2}, M_{2}, s_{2}\right)$. Through the symmetry that follows from Proposition 7 , we also find the inequality $T_{q}^{s_{2}}\left(\tau_{2}\right) \leq T_{q}^{s_{1}}\left(\tau_{1}\right)$. The proof is completed.

Remark 4. From the previous theorem, it follows that if $T_{q}^{s_{1}}\left(\tau_{1}\right) \neq T_{q}^{s_{2}}\left(\tau_{2}\right)$, then the corresponding fuzzy dynamical systems $\left(X_{1}, M_{1}, s_{1}, \tau_{1}\right),\left(X_{2}, M_{2}, s_{2}, \tau_{2}\right)$ are non-isomorphic. This means that the Tsallis entropy can be used as an instrument for distinguishing some non-isomorphic fuzzy dynamical systems.

We conclude the article with the formulation of the Kolmogorov-Sinai theorem on generators [62] (see also [11,63]) for the case of Tsallis entropy of fuzzy dynamical systems. This theorem provides a useful tool (see [62]) for calculating the entropy of dynamical systems.

Definition 13. A fuzzy partition $\delta$ of $(X, M, s)$ is said to be a generator of a fuzzy dynamical system $(X, M, s, \tau)$, if for every fuzzy partition $\alpha$ of $(X, M, s)$, there exists an integer $k>0$ such that $\alpha \prec \vee_{i=0}^{k} \tau^{i}(\delta)$.

Theorem 18. Let $\delta$ be a generator of a fuzzy dynamical system $(X, M, s, \tau)$. Then $T_{q}^{s}(\tau)=T_{q}^{s}(\tau, \delta)$.

Proof. By the assumption, for every fuzzy partition $\alpha$ of $(X, M, s)$, there exists an integer $k>0$ such that $\alpha \prec \vee_{i=0}^{k} \tau^{i}(\delta)$. Hence, according to Theorems 16 and 15, we obtain:

$$
T_{q}^{s}(\tau, \alpha) \leq T_{q}^{s}\left(\tau, \vee_{i=0}^{k} \tau^{i}(\delta)\right)=T_{q}^{s}(\tau, \delta),
$$


for every fuzzy partition $\alpha$ of $(X, M, s)$. It follows that:

$$
T_{q}^{s}(\tau)=\sup \left\{T_{q}^{s}(\tau, \alpha) ; \alpha \text { is a fuzzy partition of }(X, M, s)\right\} \leq T_{q}^{s}(\tau, \delta) .
$$

Since the converse inequality is immediate, this proves the claim.

\section{Conclusions}

The main objective of this article was to construct a mathematical model for the Tsallis entropy of fuzzy dynamical systems. The results are given in Sections 3 and 4.

In Section 3, we introduced the notion of the Tsallis entropy $T_{q}^{s}(\alpha)$ of order $q$, where $q \in(0,1) \cup$ $(1, \infty)$, of a fuzzy partition $\alpha$ and its conditional version. It was shown that the proposed concepts are consistent, in the case of the limit of $q$ going to 1 , with the Shannon entropy expressed in nats, defined and studied in Reference [29]. Furthermore, if we insert $q=2$ into the proposed definitions, we obtain the logical entropy of fuzzy partitions defined and studied in Reference [9]. Next, we derived the fundamental properties of the proposed entropy measures. Theorem 3 states that the Tsallis entropy $T_{q}^{s}(\alpha)$ monotonically decreases with respect to $q \in(0,1) \cup(1, \infty)$. The relationship between the Tsallis entropy and the Shannon-type entropy of a fuzzy partition was provided in Remark 3. In addition, the Tsallis entropy $T_{q}^{s}(\alpha)$ was shown to be a concave function on the family of all fuzzy P-measures defined on a given fuzzy measurable space $(X, M)$. In Theorem 11, chain rules for the Tsallis entropy of fuzzy partitions were provided. As further important results, we showed that the Tsallis entropy of fuzzy partitions of order $q>1$ satisfies the properties of sub-additivity and monotonicity. As shown in Examples 4 and 5, the Tsallis entropy of order $q \in(0,1)$ does not satisfy these properties in general.

Section 4 was concerned with the mathematical modeling of Tsallis entropy in fuzzy dynamical systems. Based on the results from the previous section, we defined the Tsallis entropy of order $q>1$ of a fuzzy dynamical system, and we constructed a Kolmogorov-Sinai-type isomorphism theory for the proposed entropy measure. We showed that the Tsallis entropy is invariant under isomorphisms of fuzzy dynamical systems; we thus acquired a tool for distinguishing some non-isomorphic fuzzy dynamical systems. Finally, we provided a version of the Kolmogorov-Sinai theorem on generators for the case of the Tsallis entropy of a fuzzy dynamical system.

To illustrate the obtained results, some examples were provided. From Examples 1 and 7, it follows that the model studied in this paper generalizes the classical one in which an event is understood as an exactly defined phenomenon and partitions are defined in the context of the classical Cantor's set theory. In real life, however, we often encounter phenomena that are determined vaguely. It turns out that partitions defined using the fuzzy set theory are more appropriate for solving real problems. The obtained results could be useful in the case of experiments with vague, incomplete information. Finally, we remark that the subject of our further research will be the study of the Tsallis divergence (cf. [34]) in the fuzzy case, with the aim of generalizing our previous results concerning the Kullback-Leibler divergence given in Reference [33] to the case of the Tsallis divergence.

Funding: This research received no external funding.

Acknowledgments: The article is dedicated to the memory of Beloslav Riečan, who passed away unexpectedly on 13 August 2018 and who was its intended co-author. The article builds on ideas that had arisen during our previous collaboration. Rest in peace, our beloved friend, co-worker, and teacher.

Conflicts of Interest: The author declares no conflict of interest.

\section{References}

1. Kolmogorov, A.N. A new metric invariant of transitive dynamical systems and automorphisms of Lebesgue spaces. Dokl. Russ. Acad. Sci. 1958, 119, 861-864.

2. Sinai, Y.G. On the notion of entropy of a dynamical system. Dokl. Russ. Acad. Sci. 1959, 124, 768-771.

3. Shannon, C.E. A Mathematical Theory of Communication. Bell Syst. Tech. J. 1948, 27, 379-423. [CrossRef] 
4. Pesin, Y.B. Characteristic Lyapunov exponents and smooth ergodic theory. Russ. Math. Surv. 1977, 32, 55-114. [CrossRef]

5. Markechová, D.; Ebrahimzadeh, A.; Eslami Giski, Z. Logical entropy of dynamical systems. Adv. Differ. Equ. 2018, 2018, 70. [CrossRef]

6. Ellerman, D. An Introduction to Logical Entropy and Its Relation to Shannon Entropy. Int. J. Seman. Comput. 2013, 7, 121-145. [CrossRef]

7. Ellerman, D. Logical Information Theory: New Foundations for Information Theory. Log. J. IGPL 2017, 25, 806-835. [CrossRef]

8. Ellerman, D. Logical Entropy: Introduction to Classical and Quantum Logical Information Theory. Entropy 2018, 20, 679. [CrossRef]

9. Markechová, D.; Riečan, B. Logical Entropy of Fuzzy Dynamical Systems. Entropy 2016, 18, 157. [CrossRef]

10. Markechová, D.; Mosapour, B.; Ebrahimzadeh, A. Logical Divergence, Logical Entropy, and Logical Mutual Information in Product MV-Algebras. Entropy 2018, 20, 129. [CrossRef]

11. Mohammadi, U. The concept of logical entropy on D-posets. J. Algebraic Struct. Appl. 2016, 1, $53-61$.

12. Ebrahimzadeh, A. Logical entropy of quantum dynamical systems. Open Phys. 2016, 14, 1-5. [CrossRef]

13. Ebrahimzadeh, A. Quantum conditional logical entropy of dynamical systems. Ital. J. Pure Appl. Math. 2016, 36, 879-886.

14. Markechová, D.; Riečan, B. Logical entropy and logical mutual information of experiments in the intuitionistic fuzzy case. Entropy 2017, 19, 429. [CrossRef]

15. Ebrahimzadeh, A.; Eslami Giski, Z.; Markechová, D. Logical entropy of dynamical systems-A general model. Mathematics 2017, 5, 4. [CrossRef]

16. Ebrahimzadeh, A.; Jamalzadeh, J. Conditional logical entropy of fuzzy $\sigma$-algebras. J. Intell. Fuzzy Syst. 2017, 33, 1019-1026. [CrossRef]

17. Eslami Giski, Z.; Ebrahimzadeh, A. An introduction of logical entropy on sequential effect algebra. Indag. Math. 2017, 28, 928-937. [CrossRef]

18. Zadeh, L.A. Fuzzy Sets. Inf. Control 1965, 8, 338-358. [CrossRef]

19. Dumitrescu, D. Fuzzy measures and entropy of fuzzy partitions. J. Math. Anal. Appl. 1993, 176, 359-373. [CrossRef]

20. Dumitrescu, D. Entropy of a fuzzy dynamical system. Fuzzy Sets Syst. 1995, 70, 45-57. [CrossRef]

21. Mesiar, R. The Bayes principle and the entropy on fuzzy probability spaces. Int. J. Gen. Syst. 1991, 20, 67-72. [CrossRef]

22. Mesiar, R.; Rybárik, J. Entropy of Fuzzy Partitions-A General Model. Fuzzy Sets Syst. 1998, 99, 73-79. [CrossRef]

23. Criado, F.; Gachechiladze, T. Entropy of fuzzy events. Fuzzy Sets Syst. 1997, 88, 99-106. [CrossRef]

24. Riečan, B. An entropy construction inspired by fuzzy sets. Soft Comput. 2003, 7, 486-488.

25. Khare, M. Fuzzy $\sigma$-algebras and conditional entropy. Fuzzy Sets Syst. 1999, 102, 287-292. [CrossRef]

26. Srivastava, P.; Khare, M.; Srivastava, Y.K. m-Equivalence, entropy and F-dynamical systems. Fuzzy Sets Syst. 2001, 121, 275-283. [CrossRef]

27. Rahimi, M.; Riazi, A. On local entropy of fuzzy partitions. Fuzzy Sets Syst. 2014, 234, 97-108. [CrossRef]

28. Rahimi, M.; Assari, A.; Ramezani, F. A Local Approach to Yager Entropy of Dynamical Systems. Int. J. Fuzzy Syst. 2016, 18, 98-102. [CrossRef]

29. Markechová, D. The entropy of fuzzy dynamical systems and generators. Fuzzy Sets Syst. 1992, 48, 351-363. [CrossRef]

30. Markechová, D. Entropy of complete fuzzy partitions. Math. Slovaca 1993, 43, 1-10.

31. Markechová, D. Entropy and mutual information of experiments in the fuzzy case. Neural Netw. World 2013, 23, 339-349. [CrossRef]

32. Markechová, D.; Riečan, B. Entropy of Fuzzy Partitions and Entropy of Fuzzy Dynamical Systems. Entropy 2016, 18, 19. [CrossRef]

33. Markechová, D. Kullback-Leibler Divergence and Mutual Information of Experiments in the Fuzzy Case. Axioms 2017, 6, 5. [CrossRef]

34. Tsallis, C. Possible generalization of Boltzmann-Gibbs statistics. J. Stat. Phys. 1988, 52, 479-487. [CrossRef]

35. Havrda, J.; Charvát, F. Quantification methods of classification processes: Concept of structural alpha-entropy. Kybernetika 1967, 3, 30-35. 
36. Tsallis, C. Introduction to Nonextensive Statistical Mechanics: Approaching a Complex World; Springer: New York, NY, USA, 2009.

37. Hanel, R.; Thurner, S. Generalized Boltzmann factors and the maximum entropy principle: Entropies for complex systems. Phys. A Stat. Mech. Its Appl. 2007, 380, 109-114. [CrossRef]

38. Almeida, M.P. Generalized entropies from first principles. Phys. A Stat. Mech Its Appl. 2001, 300, $424-432$. [CrossRef]

39. Kaniadakis, G. Statistical mechanics in the context of special relativity. Phys. Rev. E 2002, 66, 056125. [CrossRef] [PubMed]

40. Naudts, J. Deformed exponentials and logarithms in generalized thermostatistics. Phys. A Stat. Mech. Its Appl. 2002, 316, 323-334. [CrossRef]

41. Tsallis, C. Generalized entropy-based criterion for consistent testing. Phys. Rev. E 1998, 58, 1442-1445. [CrossRef]

42. Alemany, P.A.; Zanette, D.H. Fractal random walks from a variational formalism for Tsallis entropies. Phys. Rev. E 1994, 49, R956-R958. [CrossRef]

43. Tsallis, C. Nonextensive thermostatistics and fractals. Fractals 1995, 3, 541-547. [CrossRef]

44. Tsallis, C.; Anteneodo, C.; Borland, L.; Osorio, R. Nonextensive Statistical mechanics and economics. Phys. A Stat. Mech. Its Appl. 2003, 324, 89-100. [CrossRef]

45. Borland, L. Long-range memory and nonextensivity in financial markets. Europhys. News 2005, 36, $228-231$. [CrossRef]

46. Pérez, D.G.; Zunino, L.; Martín, M.T.; Pérez, D.G.; Zunino, L.; Martín, M.T.; Garavaglia, M.; Plastino, A.; Rosso, O.A. Model-free stochastic processes studied with q-wavelet-based in formational tools. Phys. Lett. A 2007, 364, 259-266. [CrossRef]

47. Huang, H.; Xie, H.; Wang, Z. The analysis of VF and VT with wavelet-based Tsallis information measure. Phys. Lett. A 2005, 336, 180-187. [CrossRef]

48. Tong, S.; Bezerianos, A.; Paul, J.; Zhu, Y.; Thakor, N. Nonextensive entropy measure of EEG following brain injury from cardiac arrest. Phys. A Stat. Mech. Its Appl. 2002, 305, 619-628. [CrossRef]

49. Rosso, O.A.; Martín, M.T.; Plastino, A. Brain electrical activity analysis using wavelet-based informational tools (II): Tsallis non-extensivity and complexity measures. Phys. A Stat. Mech. Its Appl. 2003, 320, 497-511. [CrossRef]

50. Kumar, V. Kapur's and Tsalli's Entropies: A Communication System Perspective; LAP LAMBERT Academic Publishing: Saarbrucken, Germany, 2015.

51. Ramiréz-Reyes, A.; Hernández-Montoya, A.R.; Herrera-Corral, G.; Domínguez-Jiménez, I. Determining the Entropic Index $q$ of Tsallis Entropy in Images through Redundancy. Entropy 2016, 18, 299. [CrossRef]

52. Nonextensive Statistical Mechanics and Thermodynamics. Available online: http://tsallis.cat.cbpf.br/biblio. htm (accessed on 16 November 2018).

53. Riečan, B. On the product MV-algebras. Tatra Mt. Math. Publ. 1999, 16, 143-149.

54. Di Nola, A.; Dvurečenskij, A. Product MV-algebras. Mult. Valued Log. 2001, 6, 193-215.

55. Jakubík, J. On product MV algebras. Czech. Math. J. 2002, 52, 797-810. [CrossRef]

56. Markechová, D.; Riečan, B. Tsallis entropy of product MV-algebra dynamical systems. Entropy 2018, 20, 589. [CrossRef]

57. Dubois, D.; Prade, M. A review of fuzzy set aggregation connectives. Inf. Sci. 1985, 36, 85-121. [CrossRef]

58. Dvurečenskij, A. On the existence of probability measures on fuzzy measurable spaces. Fuzzy Sets Syst. 1991, 43, 173-181. [CrossRef]

59. Piasecki, K. New concept of separated fuzzy subsets. In Proceedings of the Polish Symposium on Interval and Fuzzy Mathematics, Poznan, Poland, 26-29 August 1983; Albrycht, J., Wisniewski, H., Eds.; Wydawnictwo Politechniki Poznanskiej: Poznan, Poland, 1985; pp. 193-195.

60. Piasecki, K. Probability of fuzzy events defined as denumerable additive measure. Fuzzy Sets Syst. 1985, 17, 271-284. [CrossRef]

61. Piasecki, K. Fuzzy partitions of sets. BUSEFAL 1986, 25, 52-60.

62. Walters, P. An Introduction to Ergodic Theory; Springer: New York, NY, USA, 1982.

63. Ebrahimi, M. Generators of probability dynamical systems. Differ. Geom. Dyn. Syst. 2016, 8, 90-97.

(C) 2018 by the author. Licensee MDPI, Basel, Switzerland. This article is an open access article distributed under the terms and conditions of the Creative Commons Attribution (CC BY) license (http:/ / creativecommons.org/licenses/by/4.0/). 